
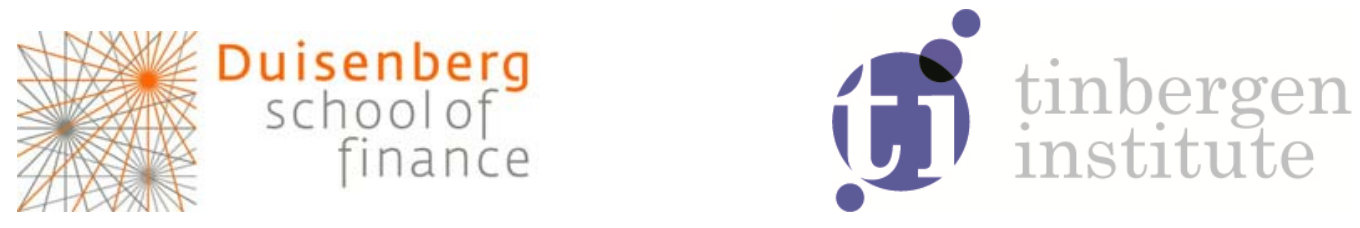

Duisenberg school of finance - Tinbergen Institute Discussion Paper

TI 11-084/2/DSF 23

\title{
Risk Measures for Autocorrelated Hedge Fund Returns
}

\author{
Antonio Di Cesarel \\ Philip A. Stork ${ }^{2}$ \\ Casper G. de Vries $^{3}$
}

I Bank of Italy;

2 VU University of Amsterdam;

3 Erasmus University Rotterdam. 
Tinbergen Institute is the graduate school and research institute in economics of Erasmus University Rotterdam, the University of Amsterdam and VU University Amsterdam.

More TI discussion papers can be downloaded at http://www.tinbergen.nl

Tinbergen Institute has two locations:

Tinbergen Institute Amsterdam

Gustav Mahlerplein 117

1082 MS Amsterdam

The Netherlands

Tel.: +31(0)205251600

Tinbergen Institute Rotterdam

Burg. Oudlaan 50

3062 PA Rotterdam

The Netherlands

Tel.: +31(0)10 4088900

Fax: +31(0)104089031

Duisenberg school of finance is a collaboration of the Dutch financial sector and universities, with the ambition to support innovative research and offer top quality academic education in core areas of finance.

DSF research papers can be downloaded at: http://www.dsf.nl/

Duisenberg school of finance

Gustav Mahlerplein 117

1082 MS Amsterdam

The Netherlands

Tel.: +31(0)20 5258579 


\title{
Risk Measures for Autocorrelated Hedge Fund Returns
}

\author{
Antonio Di Cesare* Philip A. Stork ${ }^{\dagger} \quad$ Casper G. de Vries ${ }^{\ddagger}$
}

May 2, 2011

\begin{abstract}
Standard risk metrics tend to underestimate the true risks of hedge funds because of serial correlation in the reported returns. Getmansky et al. (2004) derive mean, variance, Sharpe ratio, and beta formulae adjusted for serial correlation. Following their lead, adjusted downside and global measures of individual and systemic risks are derived. We distinguish between normally and fat tailed distributed returns and show that adjustment is particularly relevant for downside risk measures in the case of fat tails. A hedge fund case study reveals that the unadjusted risk measures considerably underestimate the true extent of individual and systemic risks.
\end{abstract}

JEL classification: G12; G23; G28.

Keywords: Hedge funds; Serial correlation; Systemic risk; VaR; Pareto distribution.

${ }^{*}$ Corresponding author: Antonio Di Cesare, Bank of Italy, Economic Outlook and Monetary Policy Department, Via Nazionale 91, 00184 Rome, Italy; Tel.: +39-06-47923943; Fax: +39-06-47923720; Email: antonio.dicesare@bancaditalia.it. The views expressed in the article are those of the author and do not involve the responsibility of the Bank of Italy.

${ }^{\dagger}$ Duisenberg School of Finance, Amsterdam, The Netherlands, and VU University Amsterdam, School of Finance and Risk Management, Amsterdam, The Netherlands.

${ }^{\ddagger}$ Duisenberg School of Finance, Amsterdam, The Netherlands, Erasmus University Rotterdam, Department of Economics, Rotterdam, The Netherlands, and Tinbergen Institute, Rotterdam, The Netherlands. 


\section{Contents}

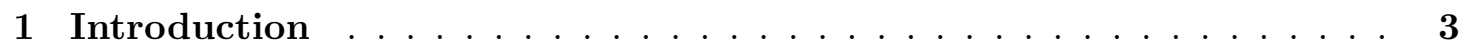

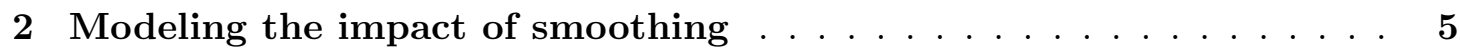

2.1 The effect of smoothing on univariate risk measures . . . . . . . 5

2.1 .1 Sharpe ratio . . . . . . . . . . . . . . . 7

2.1 .2 Value-at-risk . . . . . . . . . . . . . . . . 7

2.1 .3 Expected shortfall . . . . . . . . . . . . . . . . . 10

2.2 Smoothing effects on multivariate risk measures . . . . . . . . . . . . . 12

2.2.1 Pairwise correlation . . . . . . . . . . . . . . 12

2.2 .2 The extreme linkage measure . . . . . . . . . . . . . 13

3 Empirical methodology . . . . . . . . . . . . . . . . . . 17

3.1 Estimation of the smoothing coefficients . . . . . . . . . . . 18

3.2 Estimation of the tail index and scale parameters . . . . . . . . . . 20

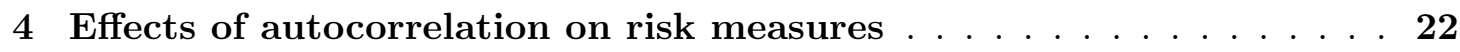

4.1 Univariate measures of risk . . . . . . . . . . . . . . . 22

4.2 Bivariate measures of risk . . . . . . . . . . . . . . . 23

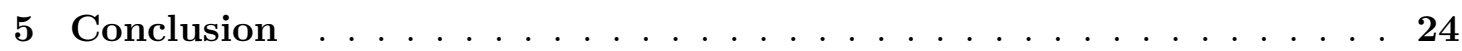

A.1 A more general framework . . . . . . . . . . . . . . . . . . . 24

A.2 Proof of proposition $7 \ldots \ldots \ldots \ldots \ldots$

A.3 Estimates of the smoothing coefficients . . . . . . . . . . 26

A.4 Small sample effects . . . . . . . . . . . . . . . . 27 


\section{Introduction}

After two decades of strong growth, hedge funds have developed into a mature and widely accepted asset class. On the back of relatively high historical returns the hedge fund industry has enjoyed a near-continuous inflow of new money, with the credit crisis period being the exception. Moreover, hedge fund risk levels are frequently reported to be lower than those of the more traditional investments in equities. These performance characteristics of hedge funds have also attracted considerable academic attention. ${ }^{2}$

One particular feature of hedge fund returns is the strong autocorrelation. Fung and Hsieh (2001), Brooks and Kat (2002), and Agarwal and Naik (2004) demonstrate that this feature invalidates standard mean-variance analysis for hedge funds. Getmansky et al. (2004) argue that the autocorrelation stems from the illiquidity of the assets held by hedge funds and the smoothing of the returns because of reporting practices. Based on a moving average representation of reported returns, they show how this process affects the Sharpe ratio (SR) and beta in a standard single index market model. As the smoothing lowers the variance of the returns and the covariance (with the market index), but leaves the mean unaffected, the standard risk measures tend to underestimate the actual risk (SR is overstated). In Chan et al. (2006), this framework is used to evaluate the systemic risk posed by hedge funds for the banking sector. Bollen and Pool (2009) use this autocorrelation structure to detect misreporting. Recently, Avramov et al. (2011) and Teo (2011) use the algorithm of Getmansky et al. (2004) to unsmooth hedge fund returns.

This paper extends the lead taken by Getmansky et al. (2004) along three dimensions. First, whereas Getmansky et al. (2004) consider two global measures of risk (SR and market beta), we broaden the scope by also evaluating popular downside measures of risk. There is considerable evidence from behavioral finance that individuals do not symmetrically treat the upside potential and the downside risk. Moreover, regulatory frameworks such as Solvency II and Basel II focus on downside risk measures.

The second dimension is the distinction between univariate (or individual) and multivariate (or systemic) measures. SR is a univariate measure of risk, while beta measures the interdependency with the market. As variance and covariance are global concepts, covering the upside and the downside, SR and beta are global risk measures. We provide for two individual and one systemic risk measures that are focused on the downside.

Finally, the third dimension adds the distinction between light tails and heavy tails. The measures considered by Getmansky et al. (2004) fully characterize the risk aspects in the case that the noise is multivariate normally distributed, that is, in the case of light tails. In practice, it is known that return distributions of most assets are heavy tailed. An example of a heavy tailed distribution is the Student's $t$-distribution. Such distributions exhibit hyperbolic or power-like decline in the tails, whereas light tailed distributions have exponential declining tails. While the SR and beta measures also

\footnotetext{
${ }^{2}$ For research on the risk and return characteristics of hedge funds, see Fung and Hsieh (1997), Ackermann et al. (1999), Agarwal and Naik (2000, 2004), Amin and Kat (2003), Morton et al. (2006), Bali et al. (2007), Eling and Schuhmacher (2007), Kosowski et al. (2007), Fung et al. (2008), Bollen and Whaley (2009), Ding et al. (2009), Sadka (2010), and Dichev and Yu (2011).
} 
apply in case of heavy tails (as long as second moments are finite), the downside risk measures do respond quite differently to smoothing depending on whether the returns are light or heavy.

More specific, what are the risk measures that we study? Apart from the univariate global SR measure considered by Getmansky et al. (2004), we also investigate the Value-at-Risk (VaR) and Expected Shortfall (ES) measures. The VaR and ES downside measures play a central role in risk management practices of the financial sector and are also sensitive to the type of tail behavior (light or fat) of the returns under consideration. As for the multivariate risk measures, we examine the correlation coefficient $\rho$, which is a global measure of risk, and a multivariate measure that focuses on the downside systemic risk. The latter measure reflects the amount of interdependence among two or more returns deep into the joint tail loss area. It exclusively picks up the extreme linkages in crisis situations and is termed the Extreme Linkage Measure (ELM). Most of these measures are well known, except perhaps ELM, which is explained in Section 2.2.2. ELM is one of the measures used to capture the systemic risk (see, e.g., International Monetary Fund, 2009, ch. 2). One of the lessons of the credit crisis is that supervisory frameworks should pay more attention to the risk exposures of the entire financial sector. The chart below summarizes our investigation for both light and heavy tails.

Risk measures analyzed in the paper

\begin{tabular}{lcc}
\hline \multirow{2}{*}{ Dimension } & \multicolumn{2}{c}{ Scope } \\
\cline { 2 - 3 } & Global & Downside \\
\hline Individual & SR & VaR, ES \\
Systemic & $\rho$ & ELM \\
\hline
\end{tabular}

After investigating how these risk measures are affected by the kind of smoothing proposed by Getmansky et al. (2004), these smoothing-adjusted risk measures are applied on four broad-based hedge fund indices between 1990 and 2009. We find that the smoothing-adjusted hedge fund investment returns indicate levels of risk that can be considerably higher than the risk measures based on reported returns would suggest. This finding holds in particular for the downside risk measures. The size of these distortions corresponds to the findings by Getmansky et al. (2004, p. 551) for the global SR measure.

Using the smoothing-adjusted economic risk measures is important for both investors trying to determine the proportion to invest in hedge funds and for investors constructing a hedge fund portfolio based on the relative risks of those funds. Correct risk measures are instrumental to prevent overpaying for an investment in hedge funds caused by overestimating the attractiveness of hedge funds. Finally, our ELM results can be of interest for policy makers and regulators who are concerned about the effects that hedge funds could have on financial stability.

The paper proceeds as follows. Section 2 models the impact of smoothing and derives the adjusted risk measures. Section 3 presents the empirical methodology. In Section 4, 
the adjusted risk measures are applied to a sample of hedge fund indices. Section 5 concludes.

\section{Modeling the impact of smoothing}

We derive how smoothing affects the risk measures introduced. Following Getmansky et al. (2004), the reported or observed returns are considered to be a weighted average of the fund's actual returns over a number of the most recent periods, including the current period. This assumption turns the observed returns into a moving average of the actual returns. Consider two hedge funds with actual returns $X_{1, t}$ and $X_{2, t}$ in period $t$. We assume that these returns adhere to a single factor market model. Thus, if $R_{t}$ is the market return in period $t$ and if $\varepsilon_{1, t}$ and $\varepsilon_{2, t}$ are idiosyncratic risk factors in period $\mathrm{t}$, then

$$
X_{i, t}=\beta_{i} R_{t}+\varepsilon_{i, t}
$$

for $i=1,2$. For the sake of the presentation we assume that both $\beta_{1}$ and $\beta_{2}$ are strictly positive constants, except when indicated otherwise, and that $R_{t}, \varepsilon_{1, t}$ and $\varepsilon_{2, t}$ are independent and identically distributed (i.i.d.) with distributions that are defined in Section 2.1. The following results are subsequently generalized to the case in which there are multiple market factors. The corresponding equations are reported in Appendix A.1. Further, the results are easily generalized to allow for negative beta that can be important for certain hedge fund strategies, such as Dedicated Short Bias. For the downside risk measures, this generalization involves considering both tails.

As in Getmansky et al. (2004, eqs. 21-23), we assume that the actual returns $X_{i, t}$ cannot be observed directly and that reported returns $S_{i, t}$ are governed by the following $M A(K)$ process

$$
\begin{aligned}
& S_{i, t}=\sum_{k=0}^{K} \theta_{i, k} X_{i, t-k}, \\
& \theta_{i, k} \in[0,1], \quad k=0, \ldots, K, \\
& \sum_{k=0}^{K} \theta_{i, k}=1,
\end{aligned}
$$

for $i=1,2$. We refer to the $M A$ coefficients $\theta_{i, k}$ as the smoothing coefficients.

\subsection{The effect of smoothing on univariate risk measures}

In this subsection, the smoothing-adjusted formulae for the univariate risk measures SR, VaR, and ES are derived. For each measure, we first treat the thin tail case followed by the fat tail case. What is meant by the thin tail case is focused on the normal distribution, which is the standard fare in finance. It provides a benchmark against which the case of heavy tails is judged. Considering the normal distribution as representative for the thin tail case is sometimes overly restrictive. For example, in the case of the SR analysis, 
we only require that the first two moments exist, so that the results for the normal case also apply to other distributions such as the uniform, the exponential, and heavy tailed distributions with the first two moments bounded. These more or less obvious generalizations are left to the reader.

A distribution is said to be (symmetrically) heavy tailed if it is regularly varying at infinity, that is to say, the tails of the distribution satisfy

$$
\lim _{t \rightarrow \infty} \frac{F(-t x)}{F(-t)}=\lim _{t \rightarrow \infty} \frac{1-F(t x)}{1-F(t)}=x^{-\alpha},
$$

for all $x>0$ and some $\alpha>0$. Somewhat loosely formulated, this definition means that to a first order at infinity the distribution follows the Pareto distribution $\mathbb{P}(|X|>x)=x^{-\alpha}$. The tail index $\alpha$ determines how heavy the tails are, as only the moments up to $\alpha$ are bounded. For example, it is readily verified that the Student's $t$-distribution with $v$ degrees of freedom has regularly varying tails with $\alpha=v$. Moreover, we will assume that the following first order condition holds

$$
\mathbb{P}(|X|>x)=2 A x^{-\alpha}+o\left(x^{-\alpha}\right),
$$

where $A>0$, that is, Eq. (5) also permits $A$ not to be constant (but requires slow variation).

To derive the implications of the $M A(K)$ process for our risk measures, we need to know the distribution of the convolution of the random variables in Eq. (2). How to do this exercise for the normal distribution is well known as the square root rule. For example, assuming that the market factor $R \sim N\left(\mu_{R}, \sigma_{R}\right)$ and the idiosyncratic factor $\varepsilon \sim N\left(\mu_{\varepsilon}, \sigma_{\varepsilon}\right)$ in Eq. (1), it follows (omitting subindices $i$ whenever no confusion is possible)

$$
X \sim N\left(\beta \mu_{R}+\mu_{\varepsilon},\left(\beta^{2} \sigma_{R}^{2}+\sigma_{\varepsilon}^{2}\right)^{1 / 2}\right) .
$$

For the case of heavy tailed distributions there does not generally exist such a simple rule. For our purposes, however, it suffices to know what happens to the tail probabilities under summation, which is a considerably simpler problem. To derive our results, we make use of the celebrated convolution theorem by Feller (1971, ch. VIII.8). The flavor of the convolution theorem is demonstrated for the case of the single index model from Eq. (1). Suppose, for example, that both the market factor $R$ and the idiosyncratic risk $\varepsilon$ are Student's $t$-distributed with $v$ degrees of freedom and hence satisfy Eq. (6). Then, the Feller convolution result holds that

$$
\lim _{t \rightarrow \infty} \frac{\mathbb{P}(X \leq-t)}{\left(\beta^{v}+1\right) A t^{-v}}=1 .
$$

It transpires that one can just add the tails if these are of equal order, provided that one scales the weights with the tail index. If the tail indices are unequal, then the tail with the lowest index dominates the sum. Note that the convolution changes the scale factor, but leaves the power $v$ unaffected. In other words, if one studies the convolution of two independent heavy tail distributed random variables with the same tail index at large 
quantiles, then it suffices to take the sum of the scales divided by the quantile to the power of tail index. To a first order, all mass in the plane concentrates along the axes and this property determines the sum. With these preparations at hand, we turn to the specific measures and investigate the effects of the moving average feature of reported returns.

\subsubsection{Sharpe ratio}

Given the single index model in Eq. (1) for the $M A(K)$ process in Eq. (2), and assuming that the market factor $R \sim N\left(\mu_{R}, \sigma_{R}\right)$ and the idiosyncratic factor $\varepsilon \sim N\left(\mu_{\varepsilon}, \sigma_{\varepsilon}\right)$, it readily follows that

$$
\sigma_{S}=\left(\sum_{k=0}^{K} \theta_{k}^{2}\right)^{1 / 2}\left(\beta^{2} \sigma_{R}^{2}+\sigma_{\varepsilon}^{2}\right)^{1 / 2}=\left(\sum_{k=0}^{K} \theta_{k}^{2}\right)^{1 / 2} \sigma_{X}
$$

Thus, $\sigma_{S} \leq \sigma_{X}$, as Eqs. (3) and (4) imply that $\sum_{k=0}^{K} \theta_{k}^{2} \leq 1$. On the other hand, the mean is unaffected

$$
\mu_{S}=\left(\sum_{k=0}^{K} \theta_{k}\right)\left(\beta \mu_{R}+\mu_{\varepsilon}\right)=\beta \mu_{R}+\mu_{\varepsilon}=\mu_{X} .
$$

These results are used to determine how the SR measure is affected by smoothing

$$
\mathrm{SR}_{X}=\frac{\mu_{X}-R_{f}}{\sigma_{X}} \leq \frac{\mu_{X}-R_{f}}{\sigma_{S}}=\mathrm{SR}_{S}
$$

where $R_{f}$ is the risk-free rate. In other words, as $\mathrm{SR}_{S}=\left(\sum \theta_{k}^{2}\right)^{1 / 2} \mathrm{SR}_{X}$, smoothing understates the (global) risk and hence SR is overstated. This finding is the same as Proposition 1 in Getmansky et al. (2004).

Next, consider the case that $R$ and $\varepsilon$ do not follow the normal distribution, but rather have distributions with heavy tails as defined above. Then Eq. (11) nevertheless applies as long as $\alpha>2$, meaning that the second moments are bounded. Hence, under the mild restriction that the variance is bounded, we merely corroborate Getmansky et al. (2004) for the SR measure. But things will be different for the downside risk measures.

\subsubsection{Value-at-risk}

VaR is probably the most widely used univariate measure in risk management. It has numerous applications in banking and insurance. Jorion (2007) provides an extensive overview of the VaR measure. VaR is sometimes criticized for its lack of subadditivity. Such criticism is why we also treat the globally subadditive ES measure below. Nevertheless, for the normal distribution VaR is subadditive below the mean. This result also holds, for example, for the Student's t-distribution. In fact, Daníelsson et al. (2010) show that for all fat tailed distributions, the VaR measure is subadditive in the tail area. So 
both for practical and theoretical reasons it is of interest to analyze how VaR is affected by the presence of autocorrelation in hedge fund returns.

For the random variable $Y_{t}$ with a continuous distribution, VaR with confidence level $p$ is defined as the unique real number $\operatorname{VaR}\left(Y_{t}, p\right)$ for which

$$
\mathbb{P}\left(Y_{t}>\operatorname{VaR}\left(Y_{t}, p\right)\right)=p
$$

or

$$
\operatorname{VaR}\left(Y_{t}, p\right)=\varphi_{Y_{t}}^{-1}(1-p),
$$

where $\varphi_{Y_{t}}^{-1}(x)$ is the inverse of the cumulative density function of $Y_{t}$ evaluated at $x$. Note that VaR usually is a loss return and hence a positive number. Therefore, we focus on the right hand tail of the loss distribution.

\subsubsection{Normal case}

Let $\operatorname{VaR}\left(S_{t}, p ; N\right)$ denote $\operatorname{VaR}$ at the confidence level $p$ for the reported return $S_{t}$ under the assumption that the i.i.d. random variables $R_{t}$ and $\varepsilon_{t}$ have normal distributions $R_{t} \sim N\left(\mu_{R}, \sigma_{R}\right)$ and $\varepsilon_{t} \sim N\left(\mu_{\varepsilon}, \sigma_{\varepsilon}\right)$. Under these assumptions, we have from the above that $S_{t} \sim N\left(\mu_{S}, \sigma_{S}\right)$. So that

$$
p=\mathbb{P}\left(S_{t}>\operatorname{VaR}\left(S_{t}, p ; N\right)\right)=1-\Phi\left(\frac{\operatorname{VaR}\left(S_{t}, p ; N\right)-\mu_{S}}{\sigma_{S}}\right),
$$

where $\Phi(x)$ is the standard normal cumulative distribution function evaluated at $x$. VaR is given by

$$
\operatorname{VaR}\left(S_{t}, p ; N\right)=\sigma_{S} \Phi^{-1}(1-p)+\mu_{S} .
$$

Given that $\mu_{S}=\mu_{X}$ and $\sigma_{S} \leq \sigma_{X}$, VaR calculated on the smoothed returns, $\operatorname{VaR}\left(S_{t}, p ; N\right)$, is always smaller than or equal to VaR calculated on the actual returns, $\operatorname{VaR}\left(X_{t}, p ; N\right)$. In particular, from Eqs. (9) and (15) we have the following:

Proposition 1 If the market factor $R$ and idiosyncratic risk are independently normally distributed, then VaR of the reported returns is related to VaR of the actual returns in the following way

$$
\frac{\operatorname{VaR}\left(S_{t}, p ; N\right)-\mu_{S}}{\operatorname{VaR}\left(X_{t}, p ; N\right)-\mu_{S}}=\frac{\sigma_{S}}{\sigma_{X}}=\left(\sum_{k=0}^{K} \theta_{k}^{2}\right)^{1 / 2} .
$$

Under the assumption of a normal distribution, the presence of autocorrelation in the actual hedge fund returns reduces the reported VaR by the reduction in the volatility of the returns. The square root rule applies again (cf. Eq. 11). 


\subsubsection{Heavy tails case}

Suppose now that the distributions of $R_{t}$ and $\varepsilon_{t}$ are heavy tailed as in Eq. (6). As a slight generalization, allow the scale $A$ in Eq. (6) to be different for $R$ and $\varepsilon$, as $\gamma_{R}$ and $\gamma_{\varepsilon}$, respectively. Let $\operatorname{VaR}\left(S_{t}, p ; H\right)$ denote $\operatorname{VaR}$ at confidence level $p$ for the case of heavy tailed distributions. Invoking Feller's convolution theorem gives

$$
\lim _{p \downarrow 0} \frac{\mathbb{P}\left(S_{t}>\operatorname{VaR}\left(S_{t}, p ; H\right)\right)}{\left(\gamma_{R} \beta^{\alpha}+\gamma_{\varepsilon}\right)\left(\sum_{k=0}^{K} \theta_{k}^{\alpha}\right) \operatorname{VaR}\left(S_{t}, p ; H\right)^{-\alpha}}=1 .
$$

On first order inversion, for small $p$ approximately

$$
\operatorname{VaR}\left(S_{t}, p ; H\right) \simeq\left(\frac{\gamma_{R} \beta^{\alpha}+\gamma_{\varepsilon}}{p} \sum_{k=0}^{K} \theta_{k}^{\alpha}\right)^{1 / \alpha} .
$$

Similarly, it is shown that for the actual returns

$$
\operatorname{VaR}\left(X_{t}, p ; H\right) \simeq\left(\frac{\gamma_{R} \beta^{\alpha}+\gamma_{\varepsilon}}{p}\right)^{1 / \alpha}
$$

We thus obtain the following:

Proposition 2 If the market factor and idiosyncratic factor exhibit heavy tails as in Eq. (6), then

$$
\lim _{p \downarrow 0} \frac{\operatorname{VaR}\left(S_{t}, p ; H\right)}{\operatorname{VaR}\left(X_{t}, p ; H\right)}=\left(\sum_{k=0}^{K} \theta_{k}^{\alpha}\right)^{1 / \alpha} .
$$

Proposition 2 is readily generalized to allow for negative betas, by taking the absolute value of beta.

Given that Eqs. (3) and (4) imply $\sum_{k=0}^{K} \theta_{k}^{\alpha} \leq 1$ for any $\alpha \geq 1$, VaR calculated on smoothed returns deep into the tail area is always smaller than or equal to VaR calculated on actual returns. The latter condition just requires that the mean is bounded. In case of the Cauchy distribution, which has $\alpha=1$, VaR calculated on smoothed returns is equal to VaR calculated on actual returns.

As the first derivative of $\left(\sum \theta_{k}^{\alpha}\right)^{1 / \alpha}$ in Eq. (20) with respect to the tail index $\alpha$ is negative, this result implies that the presence of autocorrelation affects the smoothed returns' VaR relatively less when the reported return distribution has fatter tails. In general, for any given value of the tail index $\alpha>1$, VaR of the smoothed returns $\operatorname{VaR}\left(S_{t}, p ; H\right)$ is minimized when the smoothing coefficients $\theta_{k}$ equal $1 /(K+1)$ for all $k=$ $0, \ldots, K$. In that case, the current and past true economic returns are equally weighted and together make up the reported returns. As a result, the ratio of $\operatorname{VaR}\left(S_{t}, p ; H\right)$ to $\operatorname{VaR}\left(X_{t}, p ; H\right)$ equals $(K+1)^{\frac{1-\alpha}{\alpha}}$. For $K=2$ and $\alpha=3$ this finding implies, for instance, that the reported VaR could be equal to less than half of the true VaR. 
Finally, note that the term on the left-hand side of Eq. (20) for the heavy tail case looks exactly like the correction term in Eq. (15) for the normal case, but with 2 replaced by $\alpha$. This result implies that the correction term for the heavy tail case is always smaller than the correction term for the normal case as long as $\alpha>2$. In other words, the impact of smoothing is larger in the heavy tail case than in the normal case.

\subsubsection{Expected shortfall}

Artzner et al. (1999) argue that monotonicity, subadditivity, homogeneity and translation invariance are desirable properties for a risk measure. VaR satisfies three of the four criteria, but can fail subadditivity. For this reason Artzner et al. (1999) advance the ES measure as it satisfies all four criteria (provided that $\alpha>1$ in the heavy tail case). For a given loss return threshold $y$, the ES measure is the conditional expectation

$$
\operatorname{ES}\left(Y_{t}, y\right)=\mathbb{E}\left[Y_{t} \mid Y_{t}>y\right] .
$$

In general, the ES measure is difficult to compute for the convolution induced by the $M A(K)$ process. Fortunately, for the normal case and the heavy tail case at sufficiently large $y$, we obtain explicit results.

\subsubsection{Normal case}

Using the previous assumptions, Eqs. (9) and (10) give ES as

$$
\operatorname{ES}\left(S_{t}, y ; N\right)=\frac{\int_{y}^{\infty} x e^{-\frac{1}{2}\left(\frac{x-\mu_{S}}{\sigma_{S}}\right)^{2}} \mathrm{~d} x}{\int_{y}^{\infty} e^{-\frac{1}{2}\left(\frac{x-\mu_{S}}{\sigma_{S}}\right)^{2}} \mathrm{~d} x}=\frac{\sigma_{S} \phi\left(\frac{y-\mu_{S}}{\sigma_{S}}\right)}{1-\Phi\left(\frac{y-\mu_{S}}{\sigma_{S}}\right)}+\mu_{S} .
$$

It can be shown that the first derivative of ES in Eq. (22) with respect to $\sigma_{S}$ is positive so that, given that $\sigma_{S} \leq \sigma_{X}$, ES calculated on smoothed returns is always lower than or equal to ES calculated on actual returns. Furthermore, when $y$ equals VaR that corresponds to confidence level $p$, we derive

$$
\begin{aligned}
\operatorname{ES}\left(S_{t}, \operatorname{VaR}\left(S_{t}, p ; N\right) ; N\right) & =\frac{\sigma_{S} e^{-\frac{1}{2}\left(\frac{\operatorname{VaR}\left(S_{t}, p ; N\right)-\mu_{S}}{\sigma_{S}}\right)^{2}}}{p \sqrt{2 \pi}}+\mu_{S} \\
& =\frac{e^{-\frac{1}{2}\left(\Phi^{-1}(1-p)\right)^{2}}}{p \sqrt{2 \pi} \Phi^{-1}(1-p)}\left(\operatorname{VaR}\left(S_{t}, p ; N\right)-\mu_{S}\right)+\mu_{S} .
\end{aligned}
$$

The last equation shows that ES is proportional to VaR. Given this proportionality result, it is not so surprising that the following is obtained:

Proposition 3 If the market factor $R$ and the idiosyncratic risk are independently normally distributed, then ES of the reported returns is related to ES of the actual returns as follows

$$
\frac{\operatorname{ES}\left(S_{t}, \operatorname{VaR}\left(S_{t}, p ; N\right) ; N\right)-\mu_{S}}{\operatorname{ES}\left(X_{t}, \operatorname{VaR}\left(X_{t}, p ; N\right) ; N\right)-\mu_{S}}=\frac{\sigma_{S}}{\sigma_{X}}=\left(\sum_{k=0}^{K} \theta_{k}^{2}\right)^{1 / 2} .
$$


As in the case of VaR, we find that after the correction for the mean, the smoothed ES is proportional to the actual ES. The presence of autocorrelation in the reported hedge fund returns reduces the estimated ES, and the reduction is proportional to the ratio of the two volatility estimates.

\subsubsection{Heavy tails case}

Under the same assumptions as before, we have that

$$
\lim _{x \rightarrow \infty} \frac{\mathbb{P}\left(S_{t}>x\right)}{\left(\gamma_{R} \beta^{\alpha}+\gamma_{\varepsilon}\right)\left(\sum_{k=0}^{K} \theta_{k}^{\alpha}\right) x^{-\alpha}}=1 .
$$

If the distribution function is monotonic in the tail area, it holds furthermore that the density satisfies the following asymptotic expansion (see Bingham et al., 1987)

$$
\lim _{x \rightarrow \infty} \frac{f_{S_{t}}(x)}{\alpha\left(\gamma_{R} \beta^{\alpha}+\gamma_{\varepsilon}\right)\left(\sum_{k=0}^{K} \theta_{k}^{\alpha}\right) x^{-\alpha-1}}=1 .
$$

Hence, for a sufficiently large threshold $y$ and if $\alpha>1$, the ES measure is approximately equal to

$$
\operatorname{ES}\left(S_{t}, y ; H\right) \simeq \frac{\alpha\left(\gamma_{R} \beta^{\alpha}+\gamma_{\varepsilon}\right)\left(\sum_{k=0}^{K} \theta_{k}^{\alpha}\right) \int_{y}^{\infty} x x^{-\alpha-1} \mathrm{~d} x}{\left(\gamma_{R} \beta^{\alpha}+\gamma_{\varepsilon}\right)\left(\sum_{k=0}^{K} \theta_{k}^{\alpha}\right) y^{-\alpha}}=\frac{\alpha}{\alpha-1} y .
$$

This result shows that ES is independent of the smoothing coefficients $\theta_{k}$ and of the sensitivity to the market risk $\beta$, as well as of the scale parameters $\gamma_{R}$ and $\gamma_{\varepsilon}$. The reason for this independence is that the scale parameters $\gamma_{R}$ and $\gamma_{\varepsilon}$ affect the expected value of the exceedances and the probability of exceeding the threshold in the same proportion. As a result, both effects cancel each other out. For heavy tails, ES is therefore invariant to smoothing of the returns as well as to the extent to which the returns are sensitive to movements of the stock market.

In the event that $y=\operatorname{VaR}\left(S_{t}, p ; H\right)$, we obtain from Eq. (27) that

$$
\operatorname{ES}\left(S_{t}, \operatorname{VaR}\left(S_{t}, p ; H\right) ; H\right) \simeq \frac{\alpha}{\alpha-1} \operatorname{VaR}\left(S_{t}, p ; H\right)
$$

Daníelsson et al. (2006) already obtained the analogous result for the unsmoothed returns. In Eq. (28), ES does depend on the smoothing coefficients, the market exposure, and the scale parameters. The reason for this dependence is that the VaR depends on these parameters as well (cf. Eq. 18). Consequently, the properties of ES exactly match those of the VaR. In particular, we have:

Proposition 4 If the market factor and idiosyncratic factor exhibit heavy tails as in Eq. (6), then

$$
\lim _{p \downarrow 0} \frac{\operatorname{ES}\left(S_{t}, \operatorname{VaR}\left(S_{t}, p ; H\right) ; H\right)}{\operatorname{ES}\left(X_{t}, \operatorname{VaR}\left(X_{t}, p ; H\right) ; H\right)}=\left(\sum_{k=0}^{K} \theta_{k}^{\alpha}\right)^{1 / \alpha}
$$


This last result shows that ES calculated on smoothed returns is always smaller than or equal to ES calculated on actual returns. This result mimics the one found for the VaR metric in Eq. (20).

To conclude, both in the case of normally distributed returns and in case of heavy tails, the VaR and ES measures are proportional to each other and are similarly affected by the smoothing because of reporting.

\subsection{Smoothing effects on multivariate risk measures}

In this subsection, two systemic risk measures are investigated. Getmansky et al. (2004) already consider how the estimate of the market beta for the single index model will be reduced because of smoothing. As their results apply for the normal case and the heavy tail case as long as $\alpha>2$, those results are not reproduced here. Instead, we focus on the correlation coefficient $\rho$ and the downside systemic risk measure ELM.

\subsubsection{Pairwise correlation}

We investigate the effects of smoothing on the correlation between the reported returns $\left(S_{1, t}, S_{2, t}\right)$ of two hedge funds. The case of a hedge fund and an equity index would be similar to the case of beta studied by Getmansky et al. (2004) and is therefore left to the reader. The correlation is defined as

$$
\rho\left(S_{1, t}, S_{2, t}\right)=\frac{\operatorname{Cov}\left(S_{1, t}, S_{2, t}\right)}{\left(\operatorname{Var}\left(S_{1, t}\right) \operatorname{Var}\left(S_{2, t}\right)\right)^{1 / 2}} .
$$

Assume, as before, that the market return $R_{t}$ and the idiosyncratic risk factors $\varepsilon_{1, t}, \varepsilon_{2, t}$ are i.i.d. and independent at any point in time, with variances given by, respectively, $\sigma_{R}^{2}$, $\sigma_{\varepsilon_{1}}^{2}$, and $\sigma_{\varepsilon_{2}}^{2}$. In this rather general framework only the second moments of the relevant random variables are required to exist. Standard results imply

$$
\rho\left(X_{1, t}, X_{2, t}\right)=\frac{\beta_{1} \beta_{2} \sigma_{R}^{2}}{\left(\left(\beta_{1}^{2} \sigma_{R}^{2}+\sigma_{\varepsilon_{1}}^{2}\right)\left(\beta_{2}^{2} \sigma_{R}^{2}+\sigma_{\varepsilon_{2}}^{2}\right)\right)^{1 / 2}} .
$$

For the reported hedge fund returns we have

$$
\begin{gathered}
\operatorname{Cov}\left(S_{1, t}, S_{2, t}\right)=\beta_{1} \beta_{2} \sigma_{R}^{2} \sum_{k=0}^{K} \theta_{1, k} \theta_{2, k}=\operatorname{Cov}\left(X_{1, t}, X_{2, t}\right) \sum_{k=0}^{K} \theta_{1, k} \theta_{2, k}, \\
\rho\left(S_{1, t}, S_{2, t}\right)=\frac{\sum_{k=0}^{K} \theta_{1, k} \theta_{2, k}}{\left(\left(\sum_{k=0}^{K} \theta_{1, k}^{2}\right)\left(\sum_{k=0}^{K} \theta_{2, k}^{2}\right)\right)^{1 / 2}} \frac{\beta_{R}^{2}}{\left(\left(\beta_{1}^{2} \sigma_{R}^{2}+\sigma_{\varepsilon_{1}}^{2}\right)\left(\beta_{2}^{2} \sigma_{R}^{2}+\sigma_{\varepsilon_{2}}^{2}\right)\right)^{1 / 2}} .
\end{gathered}
$$

To compare $\rho\left(X_{1, t}, X_{2, t}\right)$ from Eq. (31) with $\rho\left(S_{1, t}, S_{2, t}\right)$ from Eq. (33), recall the Cauchy-Schwarz inequality which holds that for real numbers $\theta_{1, k}$ and $\theta_{2, k}, k=0, \ldots, K$

$$
\left(\sum_{k=0}^{K} \theta_{1, k} \theta_{2, k}\right)^{2} \leq\left(\sum_{k=0}^{K} \theta_{1, k}^{2}\right)\left(\sum_{k=0}^{K} \theta_{2, k}^{2}\right) .
$$


This result is used to show:

Proposition 5 Suppose that the second moments of the factors of the market model are bounded. Then for the correlation coefficients

$$
\rho\left(S_{1, t}, S_{2, t}\right)=\frac{\sum_{k=0}^{K} \theta_{1, k} \theta_{2, k}}{\left(\left(\sum_{k=0}^{K} \theta_{1, k}^{2}\right)\left(\sum_{k=0}^{K} \theta_{2, k}^{2}\right)\right)^{1 / 2}} \rho\left(X_{1, t}, X_{2, t}\right) \leq \rho\left(X_{1, t}, X_{2, t}\right) .
$$

Note that the correlation calculated on the reported returns $\rho\left(S_{1, t}, S_{2, t}\right)$ equals the correlation calculated on the actual returns $\rho\left(X_{1, t}, X_{2, t}\right)$ if the actual returns of the two hedge funds show exactly the same pattern of autocorrelation, thus when $\theta_{1, k}=\theta_{2, k}$ for all $k$. Except for this rather exceptional case, the Cauchy-Schwarz inequality implies that the correlation calculated on the smoothed returns is always strictly smaller than the correlation calculated on the actual returns. Therefore, the correlation calculated on reported returns is likely to underestimate the true correlation calculated on actual returns. $^{3}$

\subsubsection{The extreme linkage measure}

For policy makers and risk managers, it is of utmost interest to understand the dependence of financial institutions when extreme events occur. Although the behavior under normal market circumstances is also of interest, most insight is gained when the greatest shocks occur. Contingency planning and hedging are most relevant when the markets and institutions are in extreme turmoil. The correlation measure is often criticized as an inappropriate measure of dependence as it is well-known that it performs well for normally distributed variables but much less so when returns are heavy tailed. The concept of correlation is very much tied to the specifics of the multivariate normal distribution but, from the perspective of systemic risk, it tends to give too much weight to observations in the middle of the distribution. For example, suppose that $Q$ and $W$ are two i.i.d. asset classes and that a hedge fund is long in both exposures while a second hedge fund is long in $Q$ and short in $W$ (such as in a market neutral fund). Then the portfolios $Q+W$ and $Q-W$ have zero correlation and would be independent under normality. However, in the case of the Student's $t$-distribution the two portfolios are dependent (albeit uncorrelated) because of the outliers of the two portfolios that line up along the two diagonals. We refer to Bae et al. (2003) for a clear explanation of the shortcomings of the pairwise correlation measure. Therefore, to study the systemic dependence of hedge fund returns requires turning to another measure.

ELM is a nonparametric measure of dependence based on Extreme Value Theory (EVT). It was introduced by Huang (1992) and has been applied in several empirical studies of systemic risk (see, e.g., Hartmann et al., 2004; Straetmans et al., 2008). ELM

\footnotetext{
${ }^{3}$ If the smoothing coefficients are allowed to be negative, there could also be the extreme case in which $\sum_{k=0}^{K} \theta_{1, k} \theta_{2, k}=0$, so that $\rho\left(S_{1, t}, S_{2, t}\right)=0$ irrespective of the value of $\rho\left(X_{1, t}, X_{2, t}\right)$. Hence, the reported returns would always appear to be uncorrelated even if, for example, the correlation between the actual returns equals one.
} 
is defined as the probability that both hedge funds face losses above a threshold $s$, given that at least one of the funds faces a loss in excess of that same threshold $s$

$$
\operatorname{ELM}\left(S_{1, t}, S_{2, t} ; s\right)=\frac{\mathbb{P}\left(S_{1, t}>s, S_{2, t}>s\right)}{1-\mathbb{P}\left(S_{1, t} \leq s, S_{2, t} \leq s\right)} .
$$

For theoretical purposes, ELM is evaluated in the limit as $s$ tends to infinity

$$
\operatorname{ELM}\left(S_{1, t}, S_{2, t}\right)=\lim _{s \rightarrow \infty} \operatorname{ELM}\left(S_{1, t}, S_{2, t} ; s\right) .
$$

EVT then shows that the value obtained has (empirical) relevance at finite levels, as long as $s$ is very large. If desired, the threshold levels can be easily scaled differently to account for differences in capital or size of the institution.

It is easy to show that this measure equals one minus the expected number of hedge funds that are stressed, $n$, given that at least one of the hedge funds is stressed

$$
\begin{aligned}
\mathbb{E}[n \mid n \geq 1]= & 1 \frac{\mathbb{P}\left(S_{1, t}>s, S_{2, t} \leq s\right)+\mathbb{P}\left(S_{1, t} \leq s, S_{2, t}>s\right)}{1-\mathbb{P}\left(S_{1, t} \leq s, S_{2, t} \leq s\right)} \\
& +2 \frac{\mathbb{P}\left(S_{1, t}>s, S_{2, t}>s\right)}{1-\mathbb{P}\left(S_{1, t} \leq s, S_{2, t} \leq s\right)} \\
= & \frac{\mathbb{P}\left(S_{1, t}>s\right)+\mathbb{P}\left(S_{2, t}>s\right)}{1-\mathbb{P}\left(S_{1, t} \leq s, S_{2, t} \leq s\right)} \\
= & 1+\operatorname{ELM}\left(S_{1, t}, S_{2, t} ; s\right) .
\end{aligned}
$$

In fact, it is shown that $\mathbb{E}[n \mid n \geq 1]$ readily applies to higher dimensions, by extending Eq. (38). In the bivariate case, moreover, ELM can also be expressed as

$$
\operatorname{ELM}\left(S_{1, t}, S_{2, t} ; s\right)=\frac{\mathbb{P}\left(\min \left(S_{1, t}, S_{2, t}\right)>s\right)}{\mathbb{P}\left(\max \left(S_{1, t}, S_{2, t}\right)>s\right)},
$$

where the variables $\min \left(S_{1, t}, S_{2, t}\right)$ and $\max \left(S_{1, t}, S_{2, t}\right)$ denote the minimum and the maximum values of the variables $S_{1, t}$ and $S_{2, t}$. By counting the number of excesses in the numerator and denominator of Eq. (39) at high levels of $s$, a simple nonparametric count estimator of ELM is obtained.

\subsubsection{Normal case}

Given the previous assumptions for the single index model, the $X_{1, t}$ and $X_{2, t}$ are multivariate normally distributed with correlation $\rho$ and standard deviations $\sigma_{1}$ and $\sigma_{2}$, respectively. To derive ELM, we adopt the proof of Sibuya (1960). Note that by elementary manipulations

$$
\begin{aligned}
\mathbb{E}[n \mid n \geq 1] & =\frac{\mathbb{P}\left(S_{1, t}>s\right)+\mathbb{P}\left(S_{2, t}>s\right)}{1-\mathbb{P}\left(S_{1, t} \leq s, S_{2, t} \leq s\right)} \\
& =\frac{1}{1-\frac{\mathbb{P}\left(S_{1, t}>s, S_{2, t}>s\right)}{\mathbb{P}\left(S_{1, t}>s\right)+\mathbb{P}\left(S_{2, t}>s\right)}} \\
& \leq \frac{1}{1-\frac{\mathbb{P}\left(S_{1, t}+S_{2, t}>2 s\right)}{\mathbb{P}\left(S_{1, t}>s\right)+\mathbb{P}\left(S_{2, t}>s\right)}}
\end{aligned}
$$


as the line $S_{1, t}+S_{2, t}=2 s$ cuts the slab $\left(S_{1, t}>s, S_{2, t}>s\right)$ from below. Note that $\left(S_{1, t}+S_{2, t}\right) / 2$ has variance $\left(\sigma_{1}^{2}+\sigma_{2}^{2}+2 \sigma_{1} \sigma_{2} \rho\right) / 4$, which is strictly smaller than $\max \left(\sigma_{1}^{2}, \sigma_{2}^{2}\right)$ as long as $\rho \neq 1$. The classical Laplace's tail expansion of a standard normal distribution $\Phi(s)$ with density $\phi(s)$ holds that, for large $s, 1-\Phi(s) \simeq \phi(s) / s$. It then follows that

$$
\lim _{s \rightarrow \infty} \frac{\mathbb{P}\left(\left(S_{1, t}+S_{2, t}\right) / 2>s\right)}{\mathbb{P}\left(S_{1, t}>s\right)+\mathbb{P}\left(S_{2, t}>s\right)}=0,
$$

as the rate of the exponential decay of the density of the sum (divided by two), dictated by the inverse of its variance, is greater than the rate of the exponential decay of at least one of the individual probabilities. Hence, $\mathbb{E}[n \mid n \geq 1]=1$ for large $s$ and $\operatorname{ELM}\left(S_{1, t}, S_{2, t}\right)=0$. As the proof does not depend on the particular values of the variances and correlation, it immediately follows that $\operatorname{ELM}\left(X_{1, t}, X_{2, t}\right)=0$ as well. In summary:

Proposition 6 In the case of normally distributed returns, the correlation measure summarizes the interdependency and is reduced because of smoothing. ELM tends to be nondiscriminatory and is not affected by smoothing.

Thus in the case of light tails, ELM is uninformative, as it does not depend on the particular values of the variances and the correlation, nor does it hinge on the moving average parameters. ${ }^{4}$ The global correlation measure is more informative in the case of light tails. ELM simply pierces too deeply into tails. This outcome is in sharp contrast with the case of fat tails.

\subsubsection{Heavy tails case}

For the heavy tail case, assume again that

$$
\lim _{s \rightarrow \infty} \frac{\mathbb{P}\left(R_{t}>s\right)}{\gamma_{R} s^{-\alpha}}=\lim _{s \rightarrow \infty} \frac{\mathbb{P}\left(\varepsilon_{1, t}>s\right)}{\gamma_{\varepsilon_{1}} s^{-\alpha}}=\lim _{s \rightarrow \infty} \frac{\mathbb{P}\left(\varepsilon_{2, t}>s\right)}{\gamma_{\varepsilon_{2}} s^{-\alpha}}=1,
$$

where the scale parameters $\gamma_{R}, \gamma_{\varepsilon_{1}}, \gamma_{\varepsilon_{2}}$ are strictly positive constants. For values of the threshold $s$ high enough such that Feller's theorem provides for a good approximation for the convolution of our random variables, we have

$$
\begin{gathered}
\mathbb{P}\left(X_{i, t}>s\right) \simeq\left(\beta_{i}^{\alpha} \gamma_{R}+\gamma_{\varepsilon_{i}}\right) s^{-\alpha} \\
1-\mathbb{P}\left(X_{1, t} \leq s, X_{2, t} \leq s\right) \simeq\left(\gamma_{\varepsilon_{1}}+\gamma_{\varepsilon_{2}}+\left(\max \left(\beta_{1}, \beta_{2}\right)\right)^{\alpha} \gamma_{R}\right) s^{-\alpha} .
\end{gathered}
$$

The first expression in Eq. (43) is a straightforward application of Feller's theorem as used in Eq. (17) and explained above in Eq. (8). With equal tail indices, Feller's theorem basically states that sufficiently far from the origin the probability mass above

\footnotetext{
${ }^{4}$ This conclusion is true also in the case in which $\rho=1$. It is easy to check that even in that special case, the previous results still hold as long as $\sigma_{1} \neq \sigma_{2}$. Moreover, if $\sigma_{1}=\sigma_{2}$ then $\mathbb{E}[n \mid n \geq 1]=2$ for large $s$ and $\operatorname{ELM}\left(S_{1, t}, S_{2, t}\right)=1$. These results imply that ELM is uninformative anyway.
} 
a hyperplane or a multidimensional figure of any shape that separates the space into two parts, is determined by the mass along the axes above this hyperplane or multidimensional figure. So one can just add the univariate probability mass loaded on these axes above the points where the figure cuts the axes.

For the second expression in Eq. (44), the three axes with univariate probability mass are the two idiosyncratic risk factors and the one with market risk. The boundary of $1-\mathbb{P}\left(X_{1, t} \leq s, X_{2, t} \leq s\right)$ is a pyramid shaped figure with respective boundaries of $\gamma_{\epsilon_{1}} s^{-\alpha}, \gamma_{\epsilon_{2}} s^{-\alpha}$ and $\max \left(\beta_{1}, \beta_{2}\right)^{\alpha} \gamma_{R} s^{-\alpha}$. Summation then yields the right-hand side of Eq. (44).

From Eq. (38) we then have

$$
\begin{aligned}
\operatorname{ELM}\left(X_{1, t}, X_{2, t}\right) & =\frac{\beta_{1}^{\alpha} \gamma_{R}+\gamma_{\varepsilon_{1}}+\beta_{2}^{\alpha} \gamma_{R}+\gamma_{\varepsilon_{2}}}{\gamma_{\varepsilon_{1}}+\gamma_{\varepsilon_{2}}+\left(\max \left(\beta_{1}, \beta_{2}\right)\right)^{\alpha} \gamma_{R}}-1 \\
& =\frac{\left(\min \left(\beta_{1}, \beta_{2}\right)\right)^{\alpha} \gamma_{R}}{\gamma_{\varepsilon_{1}}+\gamma_{\varepsilon_{2}}+\left(\max \left(\beta_{1}, \beta_{2}\right)\right)^{\alpha} \gamma_{R}} .
\end{aligned}
$$

For the actual hedge fund returns $S_{1, t}$ and $S_{2, t}$ the following equations apply

$$
\begin{gathered}
\mathbb{P}\left(S_{i, t}>s\right)=\mathbb{P}\left(X_{i, t}>s\right) \sum_{k=0}^{K} \theta_{i, k}^{\alpha}=\left(\beta_{i}^{\alpha} \gamma_{R}+\gamma_{\varepsilon_{i}}\right) s^{-\alpha} \sum_{k=0}^{K} \theta_{i, k}^{\alpha}, \\
1-\mathbb{P}\left(S_{1, t} \leq x, S_{2, t} \leq x\right)=\sum_{k=0}^{K}\left(\theta_{1, k}^{\alpha} \gamma_{\varepsilon_{1}}+\theta_{2, k}^{\alpha} \gamma_{\varepsilon_{2}}+\left(\max \left(\beta_{1} \theta_{1, k}, \beta_{2} \theta_{2, k}\right)\right)^{\alpha} \gamma_{R}\right) s^{-\alpha},
\end{gathered}
$$

so that

$$
\begin{aligned}
\operatorname{ELM}\left(S_{1, t}, S_{2, t}\right) & =\frac{\left(\beta_{1}^{\alpha} \gamma_{R}+\gamma_{\varepsilon_{1}}\right) \sum_{k=0}^{K} \theta_{1, k}^{\alpha}+\left(\beta_{2}^{\alpha} \gamma_{R}+\gamma_{\varepsilon_{1}}\right) \sum_{k=0}^{K} \theta_{2, k}^{\alpha}}{\sum_{k=0}^{K}\left(\theta_{1, k}^{\alpha} \gamma_{\varepsilon_{1}}+\theta_{2, k}^{\alpha} \gamma_{\varepsilon_{2}}+\left(\max \left(\beta_{1} \theta_{1, k}, \beta_{2} \theta_{2, k}\right)\right)^{\alpha} \gamma_{R}\right)}-1 \\
& =\frac{\sum_{k=0}^{K}\left(\min \left(\beta_{1} \theta_{1, k}, \beta_{2} \theta_{2, k}\right)\right)^{\alpha} \gamma_{R}}{\sum_{k=0}^{K}\left(\theta_{1, k}^{\alpha} \gamma_{\varepsilon_{1}}+\theta_{2, k}^{\alpha} \gamma_{\varepsilon_{2}}+\left(\max \left(\beta_{1} \theta_{1, k}, \beta_{2} \theta_{2, k}\right)\right)^{\alpha} \gamma_{R}\right)}
\end{aligned}
$$

Note that Eq. (48) simplifies to Eq. (45) when both of the hedge funds' actual returns are smoothed in exactly the same way, that is, when $\theta_{1, k}=\theta_{2, k}$ for all $k$. In this case, as in the pairwise correlation, the measure of linkage calculated on reported returns is equal to that calculated on actual returns.

Sections 2.1.1-2.1.3 and 2.2.1 show that the presence of autocorrelation reduces SR, VaR, ES, and pairwise correlation, both in the case of normally distributed returns and in case of heavy tails. In the case of heavy tails, we have the following corresponding result for ELM (proven in Appendix A.2):

Proposition 7 Suppose that the hedge fund returns $X_{1, t}$ and $X_{2, t}$ have the same market exposure as $\beta_{1}=\beta_{2}=\beta$. Moreover, the smoothed $S_{1, t}$ and $S_{2, t}$ both follow $M A(K)$ processes, possibly with different coefficients. Then, ELM based on the reported returns is lower than the true ELM if not all smoothing coefficients are equal. 
However, if the market betas of the hedge funds are sufficiently different, then ELM of the smoothed returns can be larger than ELM of the true underlying returns. To show this outcome, consider the case in which the scale parameters $\gamma_{R}, \gamma_{\varepsilon_{1}}, \gamma_{\varepsilon_{2}}$ all equal one and $\beta_{1} \theta_{1, k}<\beta_{2} \theta_{2, k}$ for all $k$. In this case, Eq. (48) implies

$$
\operatorname{ELM}\left(S_{1, t}, S_{2, t}\right)=\frac{\beta_{1}^{\alpha} \sum_{k=0}^{K} \theta_{1, k}^{\alpha}}{\sum_{k=0}^{K} \theta_{1, k}^{\alpha}+\left(1+\beta_{2}\right) \sum_{k=0}^{K} \theta_{2, k}^{\alpha}}=\frac{\beta_{1}^{\alpha}}{1+\left(1+\beta_{2}\right) \frac{\sum_{k=0}^{K} \theta_{2, k}^{\alpha}}{\sum_{k=0}^{K} \theta_{1, k}^{\alpha}}} .
$$

Eq. (49) shows that ELM depends on the ratio $\sum \theta_{2, k}^{\alpha} / \sum \theta_{1, k}^{\alpha}$. The presence of autocorrelation can either increase or decrease the estimated ELM as compared with the no-smoothing case when $\theta_{1,0}=\theta_{2,0}=1$ and $\theta_{1, k}=\theta_{2, k}=0$ for $k=1, \ldots, K$. Therefore, we have:

Proposition 8 If the factors of the market model for two series of returns exhibit heavy tails as in Eq. (6), then it is not possible to establish ex-ante the impact of smoothing on ELM of the two series.

In summary, if the market betas of the hedge funds are very similar, then it can be expected that the reported returns induce a lower measure of systemic risk than is factual. However, in general, the sign of the impact of smoothing cannot be established a priori for ELM and the smoothing coefficients must be estimated to determine which direction the reported returns bias the systemic risk measure.

\section{$3 \quad$ Empirical methodology}

The theory developed in Section 2 is applied using data from Hedge Fund Research (HFR). HFR identifies four primary strategy classes of hedge funds: Equity Hedge, Event-Driven, Macro, and Relative Value. We use HFR's equally weighted total return indices denominated in U.S. dollars for each of these four classes, which together include around 2,000 hedge funds. We refer to HFR's web site for additional information on these indices (see www.hedgefundresearch.com). For our empirical analysis, monthly returns across the period January 1990 to August 2009 are used, which amounts to 236 months in total.

$$
<\text { Place Figure } 1 \text { around here }>
$$

Fig. 1 reports the cumulative returns of the four indices and the Standard and Poor's 500 (S\&P 500) total return index. It illustrates the main reason for the success and growth of the hedge fund industry. Over the last two decades, each of the four indices has outperformed the S\&P 500 index by showing higher returns as well as lower volatility. The most striking period is the last decade where the cumulative return of the S\&P 500 index since 1999 has been negligible, while the four hedge fund indices have delivered large positive cumulative returns. We also glean from Fig. 1 that the recent financial crisis has affected the main hedge fund indices markedly less than the S\&P 500 index. The key statistics are reported in Table 1. 
$<$ Place Table 1 around here $>$

Table 1 shows that the hedge fund indices have SRs that are four to six times larger than that of the S\&P 500 index. Table 1 also illustrates that volatility is not an appropriate risk indicator for hedge fund indices. For instance, the Relative Value index has the lowest standard deviation and the highest SR, but it has also an extremely negative skewness and a very large kurtosis. Next, Fig. 2 shows the monthly returns of the four indices.

$<$ Place Figure 2 around here $>$

The four plots in Fig. 2 highlight two other features of hedge fund returns that are particularly important when studying systemic risk. First, although returns are stable and mostly positive, outliers occasionally do occur. In most cases these outliers are negative. Second, the negative outliers tend to occur simultaneously. An example is found in August 1998, caused by the Russian crisis and the LTCM collapse. Another example of strong negative co-movement of hedge funds occurs in the last quarter of 2008, after the failure of Lehman Brothers.

This kind of extreme dependence is seen more clearly in Fig. 3 which reports a scatter plot of the returns of the Equity Hedge and Event-Driven indices.

\section{$<$ Place Figure 3 around here $>$}

In Fig. 3, the hedge fund total return series have been transformed to have uniform marginal distributions. The elliptical shape of the plot indicates that the returns of the two indices tend to move together. More important, the greater density of points in the lower-left part of the plot highlights that the most negative extreme returns of the two indices tend to occur simultaneously.

\subsection{Estimation of the smoothing coefficients}

In this subsection, the smoothing coefficients $\theta_{k}$ are estimated. Following Getmansky et al. (2004), we conduct both maximum likelihood and linear regression estimations using an $M A(2)$ smoothing model. More precise, in the maximum likelihood estimation we assume the process of observed returns to be $S_{t}=\theta_{0} X_{t}+\theta_{1} X_{t-1}+\theta_{2} X_{t-2}$, with $X_{t}$ i.i.d., $\theta_{0}+\theta_{1}+\theta_{2}=1$ and $\theta_{k} \in[0,1]$, for $k=0,1,2$. In our numerical procedures, we do not explicitly impose the additional restrictions $\theta_{1}<1 / 2$ and $\theta_{1}<1-2 \theta_{2}$, which ensure that the $M A(2)$ process is invertible (cf. Proposition 3 in Getmansky et al., 2004). However, our estimates satisfy these restrictions in all cases. Furthermore, $X_{t}$ is not required to be normally distributed, as in Getmansky et al. (2004). Nevertheless, we are still allowed to exploit the asymptotic normality of the maximum likelihood estimator (cf. Paragraph 8.8 in Brockwell and Davis, 1991).

Getmansky et al. (2004) also argue that consistent, although not efficient, estimates of the smoothing coefficients $\theta_{k}, k=0,1,2$, can be obtained by running ordinary least squares regressions of the following equation: $S_{t}=\mu+\beta\left(\theta_{0} R_{t}+\theta_{1} R_{t-1}+\theta_{2} R_{t-2}\right)+u_{t}=$ 
$\mu+\gamma_{0} R_{t}+\gamma_{1} R_{t-1}+\gamma_{2} R_{t-2}+u_{t}$, where $u_{t}=\theta_{0} \varepsilon_{t}+\theta_{1} \varepsilon_{t-1}+\theta_{2} \varepsilon_{t-2}$, with $\varepsilon_{t}$ i.i.d., $\theta_{0}+\theta_{1}+\theta_{2}=1$ and $\theta_{k} \in[0,1]$, for $k=0,1,2$. In this case, $\hat{\beta}=\hat{\gamma}_{0}+\hat{\gamma}_{1}+\hat{\gamma}_{2}$ and $\hat{\theta}_{k}=\hat{\gamma}_{k} / \hat{\beta}^{5}$ Adding additional factors to the hedge fund model could increase the model's explanatory power (see Hasanhodzic and Lo, 2007). However, the focus of our paper is to investigate the proposed methodological improvements to hedge fund risk measures, for which a direct comparison with Getmansky et al. (2004) gives the most clarity. Therefore, we use the S\&P 500 index as the only underlying factor in our regressions.

Table 2 reports the estimated smoothing coefficients for the four indices and the S\&P 500 index using both the maximum likelihood and the linear regression estimators.

$<$ Place Table 2 around here $>$

The estimates of both methods are similar. Moreover, our results are comparable with those in Getmansky et al. (2004), even though they use different indices. We glean from Table 2 that the estimated smoothing coefficients $\hat{\theta}_{1}$ and $\hat{\theta}_{2}$ are statistically different from zero for all four hedge fund indices. The only exception is parameter estimate $\hat{\theta}_{2}$ for the Macro index. Conversely, parameter estimate $\hat{\theta}_{0}$ is always statistically different from one. The linear regression estimation approach allows us to also estimate parameter $\beta$, which measures the sensitivity of the hedge fund index returns to the market index returns.

These estimates are now used to determine how smoothing affects SR. Table 1 reports that the uncorrected SRs for the Equity Hedge and Event-Driven indices equal 0.96 and 1.03, respectively. Our correction for smoothing effects takes the same approach as Proposition 1 in Getmansky et al. (2004). We use parameter values obtained through the maximum likelihood estimation method. After unsmoothing the returns, SRs drop approximately $20 \%$ and $30 \%$, respectively, to a value of 0.74 for both indices. Although results are not directly comparable, it is worth noting that this large change in SR exceeds the impact of unsmoothing found by Getmansky et al. (2004, p. 588). ${ }^{6}$

Next, two hedge fund indices are selected to illustrate the empirical relevance of our risk measurement correction methodology. Table 2 shows that the adjusted $R^{2}$ is highest for the Equity Hedge and Event-Driven indices at 0.54 and 0.58, respectively. The $R^{2}$ is much lower for the Macro and the Relative Value indices at 0.11 and 0.34 , respectively. For reasons of brevity, the empirical applications in the remainder of the paper are limited to the Equity Hedge and Event-Driven indices only.

Because hedge funds change their investment exposures frequently, it is likely that the smoothing coefficients are not constant over time. As a result the calculation of the

\footnotetext{
${ }^{5}$ To have efficient estimates, Getmansky et al. (2004) suggest using the maximum likelihood estimator. However in our case, the results are almost identical for both the OLS and the maximum likelihood estimators. Results obtained with the maximum likelihood estimator are available on request.

${ }^{6}$ One difference between our empirical exercise and Getmansky et al. (2004) is that we use data from HFR for the period 1990-2009 while Getmansky et al. (2004) use data from TASS for the period 1977-2001. Moreover, we run our estimators on indices while the other authors use data on individual funds. Finally, our SR is calculated with respect to the USD 3-month Libor rate while Getmansky et al. (2004) use a zero interest rate benchmark.
} 
risk measures could be affected as well. For this reason we also estimate the smoothing coefficients using rolling windows of 60 months. Figs. A.1 and A.2 in Appendix A.3 report our parameter estimates for both the Equity Hedge and Event-Driven indices, using the maximum likelihood as well as the linear regression approach.

We find that the coefficients tend to remain fairly stable across the sample period 1995-2009. Furthermore, the resulting parameter levels are quite similar across the two estimation methods. These results provide support to the choice of the S\&P 500 index as underlying risk factor in our estimates.

\subsection{Estimation of the tail index and scale parameters}

How does the presence of autocorrelation affect the various downside risk measures when the market return and the idiosyncratic risk factors follow a heavy tail distribution? To answer this question, we first estimate the tail index $(\alpha)$ and the scale parameters $\left(\gamma_{R}, \gamma_{\varepsilon_{1}}, \gamma_{\varepsilon_{2}}\right)$. Thereafter, VaR using Eq. (19) and ELM from Eqs. (45) and (48) are calculated.

To estimate the tail index, we use the standard Hill (1975) estimator

$$
\frac{1}{\hat{\alpha}}=\frac{1}{m} \sum_{i=1}^{m} \log \left(\frac{X_{(i)}}{X_{(m+1)}}\right),
$$

where the $X_{(i)}$ are the largest descending order statistics $X_{(1)} \geq X_{(2)} \geq \cdots \geq X_{(m)} \geq$ $X_{(m+1)} \geq X_{(m+2)} \geq \cdots \geq X_{(n)}$ of the sample of $n$ return observations $X_{1}, \ldots, X_{n}$. Parameter $m$ equals the number of extreme returns exceeding $X_{(m+1)}$, which is the threshold return level above which the Pareto approximation applies (see Jansen and de Vries, 1991; Embrechts et al., 1997).

The scale parameter $\gamma$ is estimated as

$$
\hat{\gamma}=\frac{m}{n}\left(X_{(m+1)}\right)^{\hat{\alpha}}
$$

The idea behind Eq. (51) is to approximate the probability $\mathbb{P}\left(S>X_{m+1}\right) \simeq \gamma\left(X_{m+1}\right)^{-\alpha}$ by its empirical value $m / n$.

For the market factor $R$, the estimates of its tail index and its scale parameter are obtained by inserting the order statistics of the S\&P 500 index into Eqs. (50) and (51). Before the tail index and the scale estimates for the residuals $\varepsilon_{i}$ can be obtained, the residuals themselves need to be estimated first. Accordingly, we first calculate the values

$$
\hat{u}_{t}=S_{t}-\left(\hat{\mu}+\hat{\beta}\left(\hat{\theta}_{0} R_{t}+\hat{\theta}_{1} R_{t-1}+\hat{\theta}_{2} R_{t-2}\right)\right),
$$

where $\hat{\mu}, \hat{\beta}, \hat{\theta}_{0}, \hat{\theta}_{1}, \hat{\theta}_{2}$ are estimates obtained by the linear regression method described in Section 3.1. Given our assumption that $u_{t}=\theta_{0} \varepsilon_{t}+\theta_{1} \varepsilon_{t-1}+\theta_{2} \varepsilon_{t-1}$, the residuals are obtained recursively using

$$
\hat{\varepsilon}_{t}=\left(\hat{u}_{t}-\hat{\theta}_{1} \hat{\varepsilon}_{t-1}-\hat{\theta}_{2} \hat{\varepsilon}_{t-2}\right) / \hat{\theta}_{0} .
$$


Hence, we estimate the tail indices and the scale parameters for the residuals by inserting the order statistics of the estimated residuals from Eq. (53) into the Hill estimator from Eq. (50) and the scale estimator from Eq. (51).

Given the relatively small sample size of only 236 monthly observations, we use Hill plots to determine the number of higher left tail order statistics to be used in the estimators from Eqs. (50) and (51). A Hill plot depicts the value of the Hill estimator of $\alpha$ as a function of the number $m$ of extreme returns above the threshold $X_{m+1}$. It is used to select the number of higher order statistics such that the variance and bias squared are balanced in order to minimize the mean squared error (see Embrechts et al., 1997, for a description). The Hill plots are available from the authors on request. In all cases we find that the tail index hovers around three. This value is similar to the estimates provided by Jansen and de Vries (1991) and Hyung and de Vries (2005) for individual U.S. stocks. Given that the different $\alpha$ estimates are close, $\alpha=3$ is set for both the S\&P 500 index and the residuals of the hedge-fund indices in the calculations that follow.

To estimate the scale parameters, we use a method analogous to the Hill plot, but with the $\gamma$ estimates on the vertical axis (fixing $\alpha$ at 3 ). As the plots for the tail indices turn out to be relatively stable for $m \leq 24$ (corresponding to $10 \%$ of the observations) the scale parameters are set equal to the average of their estimates obtained using Eq. (51) across all $m \leq 24$ extreme returns

$$
\hat{\gamma}=\frac{1}{24} \sum_{k=1}^{24} \frac{k}{n}\left(X_{(k+1)}\right)^{3} .
$$

For the scale parameters $\gamma_{R}$ (for the stock index), $\gamma_{\varepsilon_{1}}$ (for the idiosyncratic term of the Equity Hedge index), and $\gamma_{\varepsilon_{2}}$ (for the idiosyncratic term of the Event-Driven index), these mean estimates equal 48.0, 5.0, and 2.6, respectively.

We conclude this section with a few remarks regarding the reliability of our methodology and the results that will be presented in Section 4. Following the previous discussion, it could be argued that our approach suffers from a potential small sample bias. Usually EVT estimates are based on relatively large amounts of data. Per contrast, our sample contains only 236 observations which can leave the results vulnerable to biases. Moreover, the statistical significance of our results could be weak because of the limited number of observations available. Therefore in Appendix A.4, we investigate how the adjusted EVT estimators are affected by the small sample size. In short, two main conclusions are drawn from the Monte Carlo simulation analysis. First, the simulations show that the estimates for VaR and ELM indeed tend to be biased in small samples. Our study shows that the bias is very small for VaR and somewhat larger for ELM. In both cases, the bias tends to disappear as the sample size increases. Second, the confidence intervals tend to be fairly wide in small samples but they significantly decrease in larger samples. Hence, we conclude that our empirical estimates could be somewhat affected by the small size of the sample but any potential bias will likely be fairly small and will not affect our main findings. 


\section{Effects of autocorrelation on risk measures}

In this section, we analyze how autocorrelation affects various risk measures. As hedge funds frequently modify their investment exposures, it is likely that the smoothing coefficients change over time as well. To capture these time-varying changes, rolling windows of 60 months are used in most of the following calculations.

\subsection{Univariate measures of risk}

We start with calculating VaR. Fig. 4 reports the time series estimates of the reported and uncorrected $\operatorname{VaR}(S, p)$ as well as the true and unobservable $\operatorname{VaR}(X, p)$ for the Equity Hedge and Event-Driven indices, calculated under the hypothesis that returns are normally distributed (cf. Eq. 14). Fig. 5 depicts similar estimates, but instead of assuming normal returns, fat tailed returns are assumed (cf. Eq. 19).

\section{$<$ Place Figure 4 and Figure 5 around here $>$}

A comparison of the Figs. 4 and 5 yields a number of interesting conclusions. First, the uncorrected and corrected VaRs often increase at the same points in time. Apparently the dynamics of the two VaR series are fairly similar, although the corrected VaR shows larger jumps. It is intuitive that the estimates of VaR for unsmoothed returns exhibit larger jumps.

Second, the levels of the two measures of VaR are substantially different. On several occasions the corrected VaR exceeds the uncorrected VaR by $50 \%$ or more. For both indices the differences between the uncorrected and corrected VaR levels increase substantially in 2009. The reason for these increases is that the presence of smoothing becomes stronger at the end of the sample period. This effect is also evident in Fig. A.1, which shows that the value of $\theta_{0}$ decreases in 2008 and 2009.

Third, VaRs are markedly higher when hedge fund returns are assumed to be fat tailed in comparison with the case of a normal distribution. The levels of VaRs in Fig. 5 clearly exceed the corresponding VaRs under normality in Fig. 4. Both the corrected and uncorrected series show such an effect. The normal distribution underrepresents the mass in the tails of the returns distributions.

Finally, our fourth conclusion is that the difference between the corrected and uncorrected VaR series is much greater when the distribution is fat tailed (see, e.g., Fig. 4, Panel A, and Fig. 5, Panel A). This fourth finding illustrates the relevancy of our extension of the Getmansky et al. (2004) paper. Their paper shows the importance of adjusting the risk metrics of hedge fund returns in a mean-variance framework and how correlation and SR are affected and how they should be corrected. However, those risk metrics assume that the returns are normally distributed. In practice, the returns of most assets are fat tailed and nonnormal (see Jansen and de Vries, 1991), and we show that the Getmansky et al. (2004) framework is even more relevant in those cases.

In this subsection, the focus is on the VaR metric. However, for the other univariate risk metric, the ES, similar conclusions can be drawn. The difference between the true 
unobservable ES and its unreported counterpart mimics that of VaR (see Eqs. 27-29). Thus, also for ES it is highly relevant to adjust the fat tailed hedge fund returns for smoothing effects.

\subsection{Bivariate measures of risk}

Next, we discuss how autocorrelation impacts on the bivariate measures of risk. First, consider the correlation measure. Fig. 6 depicts the development over time of the uncorrected and corrected correlations between the two hedge fund indices. Fig. 6 shows that the impact of smoothing on correlation is almost negligible. The reason for the limited difference is that for our sample the correction term in Eq. (35) has a value close to one. Note that this correction factor can be interpreted as the raw correlation between the smoothing coefficients. Thus, the similarity in the autocorrelation structure of the Equity Hedge and Event-Driven indices (see Table 2) shows that the estimated correlation is hardly affected by the smoothing effects of the reported data.

$$
<\text { Place Figure } 6 \text { around here }>
$$

Given the presence of fat tails, a better measure for the tail dependence is ELM. Eq. (48) shows that the parameter values determine how the presence of autocorrelation affects ELM, and in particular whether the smoothing-adjusted measure is greater or smaller than its unadjusted counterpart (recall Propositions 7 and 8). Using the regression-based point estimates over the entire sample period for the smoothing coefficients $\theta$ and the exposure to the market factor $\beta$ from Table 2 and the scale parameters $\gamma$ reported above, we calculate the two ELM measures as $\operatorname{ELM}\left(S_{1, t}, S_{2, t}\right)=0.21$ and $\operatorname{ELM}\left(X_{1, t}, X_{2, t}\right)=0.31$. Thus, the true measure of systemic risk is almost $50 \%$ higher than ELM based on smoothed returns.

Fig. 7 depicts ELM estimates for the Equity Hedge and Event-Driven indices based on rolling windows of 60 months. It shows that, on average, the smoothing-adjusted ELM significantly exceeds its unadjusted counterpart, confirming our estimates based on the whole sample. The relative size of the adjustment can, at times, even exceed the $50 \%$ estimation error that we found above. For instance, at the beginning of 2005 the corrected probability of one hedge fund index being under stress given that the other index is under stress, is more than $80 \%$ higher than the uncorrected probability. Also, at the end of the sample period the corrected measure exceeds the uncorrected measure by about 55\%. Per contrast, the corrected and uncorrected correlation estimates in Fig. 6 are of a very comparable level and nearly indistinguishable.

$$
<\text { Place Figure } 7 \text { around here }>
$$

The large difference between the correlation and ELM estimates underscores the relevance of our proposed adjustments to the tail dependence metric ELM. Evidently, unsmoothing the observed hedge fund returns is especially important when studying the extreme tail dependence. This part of the distribution is of most interest to policy 
makers and risk managers because of its relevance for financial stability issues and loss prevention. Our empirical analysis illustrates the economic importance of adjusting the metrics of extreme co-movement risks to prevent autocorrelation-induced distortions. Otherwise, a potentially serious underestimation error is a likely consequence.

\section{Conclusion}

Hedge fund returns frequently exhibit a strong degree of autocorrelation. As a result, the economic risks of an investment in hedge funds are easily underestimated and investment decisions can become biased. In this paper, we extend the seminal work of Getmansky et al. (2004) on SR and market beta, by developing a number of smoothing-adjusted downside risk measures and by allowing for nonnormal fat tailed return distributions. In particular, VaR, ES for individual risk exposures, correlation coefficient, and ELM reflecting downside systemic risk, are adjusted for the autocorrelation present in reported returns. We show that the adjustment of the downside risk measures for autocorrelation is more relevant when returns are fat tailed than when they are normally distributed. A hedge fund case study reveals that the unadjusted risk measures can considerably underestimate the true extent of individual and multivariate risks. Finally, we note that, although our risk-adjustment is applied to hedge funds only, our framework can also be used to evaluate the risks of other alternative investment strategies. Investments in real estate, art, collectible stamps, and other illiquid or opaque securities are also known to exhibit strong serial correlation in the reported returns. ${ }^{7}$ Also for these assets, conventional risk measures need adjustments to correctly reflect the true level of investment risk.

\section{Appendix A}

\section{A.1 A more general framework}

In the main text we assume that the hedge funds' actual returns are a function of only one market factor. In this Appendix, we show that the results can also be generalized to multiple factor models. For examples of the use of such multifactor models, we refer to Fung and Hsieh (2004), Kosowski et al. (2007), and Teo (2011). Assume that the actual returns of the two hedge funds have the following structure

$$
X_{1, t}=\sum_{n=1}^{N} \beta_{1, n} R_{n, t}+\varepsilon_{1, t} \quad \text { and } \quad X_{2, t}=\sum_{n=1}^{N} \beta_{2, n} R_{n, t}+\varepsilon_{2, t},
$$

where the variables $R_{n, t}, n=1, \ldots, N$, denote different factors. Furthermore, the idiosyncratic risk factors $\varepsilon_{1, t}, \varepsilon_{2, t}$ and the factors $R_{n, t}$ are i.i.d. and the reported returns are smoothed according to Eqs. (2)-(4). It can be shown that the results of the previous cases are generalized into the following equations

\footnotetext{
${ }^{7}$ See, for example, Ross and Zisler (1991), Campbell (2008), and Dimson and Spaenjers (2011).
} 
- Value-at-Risk

- Normal case

$$
\operatorname{VaR}\left(S_{t}, p\right)=\sigma_{S} \Phi^{-1}(1-p) \quad \text { where } \quad \sigma_{S}^{2}=\left(\sigma_{R}^{2} \sum_{n=1}^{N} \beta_{n}^{2}+\sigma_{\varepsilon}^{2}\right) \sum_{k=0}^{K} \theta_{k}^{2} .
$$

- Heavy tail case

$$
\operatorname{VaR}\left(S_{t}, p\right)=\left(\frac{\gamma_{R} \sum_{n=1}^{N} \beta_{n}^{\alpha}+\gamma_{\varepsilon}}{p} \sum_{k=0}^{K} \theta_{k}^{\alpha}\right)^{\frac{1}{\alpha}}
$$

- Expected shortfall

- Normal case

$$
\operatorname{ES}\left(S_{t}, y\right)=\frac{\sigma_{S} \phi\left(\frac{y}{\sigma_{S}}\right)}{1-\Phi\left(\frac{y}{\sigma_{S}}\right)} \quad \text { where } \quad \sigma_{S}^{2}=\left(\sigma_{R}^{2} \sum_{n=1}^{N} \beta_{n}^{2}+\sigma_{\varepsilon}^{2}\right) \sum_{k=0}^{K} \theta_{k}^{2} .
$$

- Heavy tail case

$$
\operatorname{ES}\left(S_{t}, y\right)=\frac{\alpha}{\alpha-1} y .
$$

- Correlation

$$
\rho\left(S_{1, t}, S_{2, t}\right)=\frac{\sum_{k=0}^{K} \theta_{1, k} \theta_{2, k}}{\left(\left(\sum_{k=0}^{K} \theta_{1, k}^{2}\right)\left(\sum_{k=0}^{K} \theta_{2, k}^{2}\right)\right)^{1 / 2}} \frac{\sum_{n=1}^{N} \beta_{1, n} \beta_{2, n} \sigma_{R_{n}}^{2}}{\left(\operatorname{Var}\left(X_{1}\right) \operatorname{Var}\left(X_{2}\right)\right)^{1 / 2}},
$$

with $\operatorname{Var}\left(X_{i}\right)=\sum_{n=1}^{N} \beta_{i, n}^{2} \sigma_{R_{n}}^{2}+\sigma_{\varepsilon_{i}}^{2}, i=1,2$.

- Extreme linkage measure

$$
\operatorname{ELM}\left(S_{1, t}, S_{2, t}\right)=1+\frac{\sum_{k=0}^{K} \sum_{n=1}^{N}\left(\min \left(\beta_{1, n} \theta_{1, k}, \beta_{2, n} \theta_{2, k}\right)\right)^{\alpha} \gamma_{R}}{\sum_{k=0}^{K}\left(\theta_{1, k}^{\alpha} \gamma_{\varepsilon_{1}}+\theta_{2, k}^{\alpha} \gamma_{\varepsilon_{2}}+\sum_{n=1}^{N}\left(\max \left(\beta_{1, n} \theta_{1, k}, \beta_{2, n} \theta_{2, k}\right)\right)^{\alpha} \gamma_{R}\right)}
$$

These results facilitate the estimation of the various risk measures when the hedge fund returns are best modeled by more than one (market) factor alone. 


\section{A.2 Proof of proposition 7}

Given the equal betas, the true $\operatorname{ELM}\left(X_{1, t}, X_{2, t}\right)$ from Eq. (45) reduces to

$$
\begin{aligned}
\operatorname{ELM}\left(X_{1, t}, X_{2, t}\right) & =\frac{\beta^{\alpha} \gamma_{R}}{\gamma_{\varepsilon_{1}}+\gamma_{\varepsilon_{2}}+\beta^{\alpha} \gamma_{R}} \\
& =\frac{1}{\frac{\gamma_{\varepsilon_{1}}+\gamma_{\varepsilon_{2}}}{\beta^{\alpha} \gamma_{R}}+1} .
\end{aligned}
$$

The corresponding measure for the smoothed returns from Eq. (48) becomes

$$
\operatorname{ELM}\left(S_{1, t}, S_{2, t}\right)=\frac{\sum_{k=0}^{K}\left(\min \left(\theta_{1, k}, \theta_{2, k}\right)\right)^{\alpha}}{\frac{\gamma_{\varepsilon_{1}}}{\beta^{\alpha} \gamma_{R}} \sum_{k=0}^{K} \theta_{1, k}^{\alpha}+\frac{\gamma_{\varepsilon_{2}}}{\beta^{\alpha} \gamma_{R}} \sum_{k=0}^{K} \theta_{2, k}^{\alpha}+\sum_{k=0}^{K}\left(\max \left(\theta_{1, k}, \theta_{2, k}\right)\right)^{\alpha}} .
$$

Comparing the two measures $\operatorname{ELM}\left(X_{1, t}, X_{2, t}\right) \gtreqless \operatorname{ELM}\left(S_{1, t}, S_{2, t}\right)$ shows

$$
\begin{aligned}
\frac{\gamma_{\varepsilon_{1}}}{\beta^{\alpha} \gamma_{R}} \sum_{k=0}^{K} \theta_{1, k}^{\alpha}+\frac{\gamma_{\varepsilon_{2}}}{\beta^{\alpha} \gamma_{R}} \sum_{k=0}^{K} \theta_{2, k}^{\alpha}+\sum_{k=0}^{K}\left(\max \left(\theta_{1, k}, \theta_{2, k}\right)\right)^{\alpha} \gtreqless \\
\frac{\gamma_{\varepsilon_{1}}+\gamma_{\varepsilon_{2}}}{\beta^{\alpha} \gamma_{R}} \sum_{k=0}^{K}\left(\min \left(\theta_{1, k}, \theta_{2, k}\right)\right)^{\alpha}+\sum_{k=0}^{K}\left(\min \left(\theta_{1, k}, \theta_{2, k}\right)\right)^{\alpha},
\end{aligned}
$$

or

$$
\begin{aligned}
& \sum_{k=0}^{K}\left(\left(\max \left(\theta_{1, k}, \theta_{2, k}\right)\right)^{\alpha}-\left(\min \left(\theta_{1, k}, \theta_{2, k}\right)\right)^{\alpha}\right) \gtreqless \\
& \quad \frac{\gamma_{\varepsilon_{1}}}{\beta^{\alpha} \gamma_{R}} \sum_{k=0}^{K}\left(\left(\min \left(\theta_{1, k}, \theta_{2, k}\right)\right)^{\alpha}-\theta_{1, k}^{\alpha}\right)+\frac{\gamma_{\varepsilon_{2}}}{\beta^{\alpha} \gamma_{R}} \sum_{k=0}^{K}\left(\left(\min \left(\theta_{1, k}, \theta_{2, k}\right)\right)^{\alpha}-\theta_{2, k}^{\alpha}\right) .
\end{aligned}
$$

The elements on the left-hand side are all nonnegative (and some are strictly positive if not all smoothing coefficients are equal), while the terms on the right-hand side are all nonpositive (and some are strictly negative if not all smoothing coefficients are equal). Hence, the left-hand side is always at least as large as the right-hand side, or $\operatorname{ELM}\left(X_{1, t}, X_{2, t}\right) \geq \operatorname{ELM}\left(S_{1, t}, S_{2, t}\right)$.

\section{A.3 Estimates of the smoothing coefficients}

$<$ Place Figure A.1 around here $>$

$<$ Place Figure A.2 around here $>$ 


\section{A.4 Small sample effects}

In this Appendix, we analyze how the EVT estimators in this paper are affected by the small size of the sample. The reason for conducting this analysis is that EVT estimators are usually applied to large data samples. Per contrast, our data set is relatively small as it contains only 236 return observations.

Therefore, it is uncertain how reliable the parameter estimates in our paper are. To analyze this issue, Monte Carlo simulations of the market and idiosyncratic components of hedge funds returns are used. First, a random sample from a standard normal distribution is generated. Next, the outcomes in both the first and last deciles of the sorted return observations are replaced by the corresponding random numbers drawn from a Pareto distribution with tail index $\alpha=3$ and scale parameter $\gamma=48.0 .{ }^{8}$ The result is a random sample normally distributed in the middle of the distribution but with Pareto tails. A similar approach is used by Daníelsson and de Vries (2000). The parameter values are chosen to mimic the estimated S\&P 500 index tail behavior.

Next, the same method is applied to generate two other samples. The scale parameters are set equal to 5.0 and 2.6, respectively, which mimic the estimated series of the idiosyncratic factors. With these random samples, Eqs. (1) and (2) are used to simulate the behavior of the unobservable true returns of two hedge fund indices as well as those of the corresponding reported smoothed returns. The betas are set equal to 0.61 and 0.53 , respectively. The thetas are set equal to $0.79,0.10$, and 0.11 , respectively, for the Equity Hedge index and to $0.67,0.18$, and 0.15 for the Event-Driven index. These values equal our estimates for the two hedge fund indices at the end of the sample (cf. Fig. A.2). This procedure is repeated 5,000 times to estimate the mean, as well as confidence bands, for our parameters.

Then, linear regressions are used to estimate the market sensitivity parameter $(\beta)$ and the smoothing coefficients $(\theta)$. We use moving windows of 60 observations of our random samples of 261 observations. The length of the moving windows equals 62 . However, two observations are lost in the estimation process because of the $M A(2)$ nature of our model. Moreover, our random samples are chosen to slightly exceed the length of our data set, which has 236 observations. As a result, we have exactly 200 parameters estimates obtained from 200 moving windows of 62 observations. Our results, reported in Table A.1, show that all parameter estimates are unbiased. However, because of the small size of the sample (only 60 observations), the confidence intervals and the mean absolute errors are rather large.

We find that the Hill estimates are quite stable. Our simulations show that the Hill estimates are very close to the true values (i.e., the values used to generate the random samples). The average absolute difference found for the range between the tenth and 25th observation equals 0.64. Furthermore, the confidence intervals considerably reduce in size when the number of observations is increased. The scale parameter estimates are also unbiased and show confidence intervals that decrease in size with the number of

\footnotetext{
${ }^{8}$ We also performed our Monte Carlo simulations using Student's $t$-random variables with three degrees of freedom. The results are qualitatively similar.
} 
observations used. ${ }^{9}$

Subsequently, we calculate Hill estimates of the idiosyncratic term of the hedge fund returns, and find that this Hill plot strongly resembles the one generated for the S\&P 500 index. The reason for this similarity is that the tail index of the market index equals that of the idiosyncratic terms. Additionally, it is noted that the width of the confidence intervals found for the idiosyncratic term estimates exceeds that found for the market index. The reason is that the idiosyncratic terms are not directly observable but need to be estimated. This estimation procedure increases the statistical uncertainty. Next, the scale parameter of the idiosyncratic terms is estimated. Our unreported results are very similar to those found for the Hill estimates of the idiosyncratic terms.

Given that the tails of our systematic and idiosyncratic risk factors are Pareto distributed, we use Eqs. (19) and (45) to calculate the exact values of VaR and ELM and compare them with the Monte Carlo outcomes. Figure A.3 depicts the estimated and true values of both VaR and ELM of the Equity Hedge index. Panel A of Fig. A.3 shows that our procedure generates a minor positive bias of around $1.5 \%$ in the estimate of the true unobservable VaR. ${ }^{10}$ The bias is caused by the functional form of Eq. (19) that can be shown to be convex in $\beta$. Given that $\beta$ is imprecisely estimated around its true value in small samples, our estimate for VaR tends to be larger than the true value of $\mathrm{VaR}$ because of Jensen's inequality. Of course, the larger the sample used in the estimation, the more precise the estimate of $\beta$ and the smaller the bias. Our results show that the bias is about $0.5 \%$ when estimations are performed on samples of 150 observations and it is smaller than $0.2 \%$ on samples of 300 observations (see Table A.2). Although the bias is almost negligible even in our small samples of 60 observations, the mean absolute errors are somewhat larger and equal to about $10 \%$ of the true VaR. Similar to the case of the bias, we find that mean absolute errors are much smaller in greater samples.

Compared with the above VaR results, our findings are less favorable for ELM (see Fig. A.3, Panel B). In the ELM's case, the bias is negative and approximately $10 \%$ of the true ELM. Moreover, the mean absolute error is much larger than that for VaR, and equals around $30 \%$ of the true ELM. However, note that results of the Monte Carlo simulations further reinforce our conclusions in Section 4 for ELM. Unreported simulations show that the estimated ELM for smoothed returns is unbiased. Hence, the true difference between ELM calculated on smoothed observable returns and that calculated on true unobservable returns even exceeds the difference reported in Fig. 7. Our simulation analysis further underscores the importance of correcting the risk measures when returns are smoothed.

$$
\begin{aligned}
& <\text { Place Table A.1 around here }> \\
& <\text { Place Table A.2 around here }>
\end{aligned}
$$

\footnotetext{
${ }^{9}$ More detailed results are available from the authors.

${ }^{10} \mathrm{We}$ also tried an alternative method to estimate the true VaR by running a linear regression of a log-transformed version of Eq. (18). For this procedure the mean estimate is close to the true value although it also appears to be biased. Moreover, the confidence intervals are wider than those found through the method described above. These results are available from the authors on request.
} 
$<$ Place Figure A.3 around here $>$

\section{References}

\section{References}

Ackermann, C., McEnally, R., and Ravenscraft, D., (1999). The Performance of Hedge Funds: Risk, Return, and Incentives. Journal of Finance, 54 (3), 833-874.

Agarwal, V. and Naik, N. Y., (2000). Multi-Period Performance Persistence Analysis of Hedge Funds. Journal of Financial and Quantitative Analysis, 35, 327-342.

Agarwal, V. and Naik, N. Y., (2004). Risks and Portfolio Decisions Involving Hedge Funds. Review of Financial Studies, 17 (1), 63-98.

Amin, G. S. and Kat, H. M., (2003). Hedge Fund Performance 1990-2000: Do the Money Machines Really Add Value? Journal of Financial and Quantitative Analysis, $38,251-274$.

Artzner, P., Delbaen, F., Eber, J.-M., and Heath, D., (1999). Coherent Measures of Risk. Mathematical Finance, 9 (3), 203-228.

Avramov, D., Kosowski, R., Naik, N. Y., and Teo, M., (2011). Hedge Funds, Managerial Skill, and Macroeconomic Variables. Journal of Financial Economics, 99 (3), 672-692.

Bae, K.-H., Karolyi, A. G., and Stulz, R. M., (2003). A New Approach to Measuring Financial Contagion. The Review of Financial Studies, 16 (3), 717-763.

Bali, T. G., Gokcan, S., and Liang, B., (2007). Value at Risk and the Cross-Section of Hedge Fund Returns. Journal of Banking \& Finance, 31 (4), 1135-1166.

Bingham, N. H., Goldie, C. M., and Teugels, J. L. (1987). Regular Variation. Cambridge University Press, New York.

Bollen, N. P. B. and Pool, V. K., (2009). Do Hedge Fund Managers Misreport Returns? Evidence from the Pooled Distribution. Journal of Finance, 64 (5), 2257-2288.

Bollen, N. P. B. and Whaley, R. E., (2009). Hedge Fund Dynamics: Implications for Performance Appraisal. Journal of Finance, 64, 985-1035.

Brockwell, P. and Davis, R. (1991). Time Series: Theory and Methods. Springer-Verlag, New-York.

Brooks, C. and Kat, H. M., (2002). The Statistical Properties of Hedge Fund Index Returns and Their Implications for Investors. The Journal of Alternative Investments, $5,26-44$. 
Campbell, R., (2008). Art as a Financial Investment. The Journal of Alternative Investments, 10 (4), 64-81.

Chan, N., Getmansky, M., Haas, S., and Lo, A. W. (2006). Systemic Risk and Hedge Funds. In M. Carey and R. Stulz, editors, The Risks of Financial Institutions, pages 235-330. The University of Chicago Press, Chicago.

Daníelsson, J. and de Vries, C. G., (2000). Value-at-Risk and Extreme Returns. Annales d'Économie et de Statistique, 60, 239-270.

Daníelsson, J., Jorgensen, B. N., Sarma, M., and de Vries, C. G., (2006). Comparing Downside Risk Measures for Heavy Tailed Distributions. Economic Letters, 92 (2), 202-208.

Daníelsson, J., Jorgensen, B. N., Samorodnitsky, G., Sarma, M., and de Vries, C. G. (2010). Fat Tails, VaR and Subadditivity. Electronic copy available at http://www . RiskResearch.org (accessed March 31, 2011).

Dichev, I. D. and Yu, G., (2011). Higher Risk, Lower Returns: What Hedge Fund Investors Really Earn. Journal of Financial Economics, 100 (2), 248-263.

Dimson, E. and Spaenjers, C., (2011). Ex Post: The Investment Performance of Collectible Stamps. Journal of Financial Economics, 100 (2), 443-458.

Ding, B., Shawky, H. A., and Tian, J., (2009). Liquidity Shocks, Size and the Relative Performance of Hedge Fund Strategies. Journal of Banking \& Finance, 33 (5), 883-891.

Eling, M. and Schuhmacher, F., (2007). Does the Choice of Performance Measure Influence the Evaluation Hedge Funds? Journal of Banking \& Finance, 31, 2632-2647.

Embrechts, P., Klüppelberg, C., and Mikosch, T. (1997). Modelling Extremal Events for Insurance and Finance. Springer Verlag, Berlin.

Feller, W. (1971). An Introduction to Probability Theory and Its Applications, volume 2. John Wiley \& Sons, New York.

Fung, W. and Hsieh, D. A., (1997). Empirical Characteristics of Dynamic Trading Strategies: The Case of Hedge Funds. The Review of Financial Studies, 10, 275-302.

Fung, W. and Hsieh, D. A., (2001). The Risk in Hedge Fund Strategies: Theory and Evidence from Trend Followers. The Review of Financial Studies, 14, 313-341.

Fung, W. and Hsieh, D. A., (2004). Hedge Fund Benchmarks: A Risk-Based Approach. Financial Analysts Journal, 60 (5), 65-80.

Fung, W., Hsieh, D. A., Naik, N. Y., and Ramadorai, T., (2008). Hedge Funds: Performance, Risk, and Capital Formation. Journal of Finance, 63, 1777-1803. 
Getmansky, M., Lo, A. W., and Makarov, I., (2004). An Econometric Model of Serial Correlation and Illiquidity in Hedge Fund Returns. Journal of Financial Economics, $74(3), 529-609$.

Hartmann, P., Straetmans, S., and de Vries, C. G., (2004). Asset Market Linkages in Crisis Periods. The Review of Economics and Statistics, 86 (1), 313-326.

Hasanhodzic, J. and Lo, A. W., (2007). Can Hedge-Fund Returns be Replicated? The Linear Case. Journal of Investment Management, 5 (2), 5-45.

Hill, B. M., (1975). A Simple General Approach to Inference about the Tail of a Distribution. The Annals of Statistics, 3 (5), 1163-1174.

Huang, X. (1992). Statistics of Bivariate Extreme Values. Ph.D. thesis 22, Tinbergen Institute Research Series, Erasmus University Rotterdam.

Hyung, N. and de Vries, C. G., (2005). Portfolio Diversification Effects of Downside Risk. Journal of Financial Econometrics, 3 (1), 107-125.

International Monetary Fund (2009). Global Financial Stability Report. Responding to the Financial Crisis and Measuring Systemic Risk. World Economic and Financial Surveys.

Jansen, D. W. and de Vries, C. G., (1991). On the Frequency of Large Stock Returns: Putting Booms and Busts into Perspective. The Review of Economics and Statistics, 73 (1), 18-24.

Jorion, P. (2007). Value at Risk. The New Benchmark for Managing Financial Risk. McGraw-Hill, New York, third edition.

Kosowski, R., Naik, N. Y., and Teo, M., (2007). Do Hedge Funds Deliver Alpha? A Bayesian and Bootstrap Analysis. Journal of Financial Economics, 84 (1), 229-264.

Morton, D. P., Popova, E., and Popova, I., (2006). Efficient Fund of Hedge Funds Construction under Downside Risk Measures. Journal of Banking \& Finance, 30, 503-518.

Ross, S. A. and Zisler, R. C., (1991). Risk and Return in Real Estate. Journal of Real Estate Finance and Economics, 4 (2), 175-190.

Sadka, R., (2010). Liquidity Risk and the Cross-Section of Hedge-Fund Returns. Journal of Financial Economics, 98 (1), 54-71.

Sibuya, M., (1960). Bivariate Extreme Statistics, I. Annals of the Institute of Statistical Mathematics, 11 (3), 195-210.

Straetmans, S., Verschoor, W., and Wolff, C., (2008). Extreme US Stock Market Fluctuations in the Wake of 9/11. Journal of Applied Econometrics, 23, 17-42. 
Teo, M., (2011). The Liquidity Risk of Liquid Hedge Funds. Journal of Financial Economics, 100 (1), 24-44. 


\section{Tables and figures}

Table 1

Descriptive statistics of hedge fund index returns

All statistics are based on monthly log-returns for the period January 1990 to August 2009. Mean, standard deviation and Sharpe ratio are annualized. The Sharpe ratio is calculated using the USD 3-month Libor as the risk-free rate. The Jarque-Bera statistic tests the hypothesis of normality of the returns, which is the joint hypothesis that skewness $=0$ and kurtosis $=3$.

\begin{tabular}{lcccrrc}
\hline $\begin{array}{l}\text { Investment } \\
\text { strategy }\end{array}$ & Mean & $\begin{array}{c}\text { Standard } \\
\text { deviation }\end{array}$ & $\begin{array}{c}\text { Sharpe } \\
\text { ratio }\end{array}$ & Skewness & Kurtosis & $\begin{array}{c}\text { Jarque-Bera } \\
\text { statistic }\end{array}$ \\
\hline Equity hedge & 13.19 & 9.22 & 0.96 & -0.38 & 5.08 & $48.43^{* *}$ \\
Event-driven & 11.56 & 7.02 & 1.03 & -1.50 & 7.89 & $323.64^{* *}$ \\
Macro & 13.14 & 7.70 & 1.15 & 0.35 & 3.79 & $11.02^{*}$ \\
Relative value & 10.00 & 4.55 & 1.25 & -2.46 & 18.45 & $2,586.47^{* *}$ \\
\hline S\&P 500 & 7.54 & 15.23 & 0.21 & -0.84 & 4.74 & $57.79^{* *}$ \\
\hline
\end{tabular}

Significance levels are denoted by ${ }^{* *}$ for $1 \%$ (critical value $\left.=11.69\right)$ and ${ }^{*}$ for $5 \%$ (critical value $=$ $5.72)$.

Table 2

Estimates of the smoothing coefficients

All estimates are based on monthly log-returns for the period January 1990 to August 2009. The table shows maximum likelihood and linear regression estimates of an $M A(2)$ smoothing process $S_{t}=\theta_{0} X_{t}+\theta_{1} X_{t-1}+\theta_{2} X_{t-2}$. In the linear regression case, the underlying hypothesis is that $X_{t}$ is defined by a linear single-factor model in which the factor is the Standard and Poor's 500 (S\&P 500) index. Significance levels for the maximum likelihood estimates are calculated using the asymptotic result of Theorem 3 in Getmansky et al. (2004). The null hypothesis for $\hat{\theta}_{0}$ is that it is equal to one, those for $\hat{\theta}_{1}, \hat{\theta}_{2}$ are that they are equal to zero.

\begin{tabular}{|c|c|c|c|c|c|c|c|c|}
\hline \multirow{2}{*}{$\begin{array}{l}\text { Investment } \\
\text { strategy }\end{array}$} & \multicolumn{3}{|c|}{ Maximum likelihood } & \multicolumn{5}{|c|}{ Linear regression } \\
\hline & $\hat{\theta}_{0}$ & $\hat{\theta}_{1}$ & $\hat{\theta}_{2}$ & $\hat{\theta}_{0}$ & $\hat{\theta}_{1}$ & $\hat{\theta}_{2}$ & $\hat{\beta}$ & $\begin{array}{l}\operatorname{Adj} . \\
R^{2}\end{array}$ \\
\hline Equity hedge & $0.743^{* *}$ & $0.172^{* *}$ & $0.085^{*}$ & 0.722 & 0.154 & 0.124 & 0.587 & 0.54 \\
\hline Event-driven & $0.665^{* *}$ & $0.253^{* *}$ & $0.082^{*}$ & 0.631 & 0.277 & 0.092 & 0.480 & 0.58 \\
\hline Macro & $0.860^{*}$ & $0.144^{* *}$ & -0.004 & 0.657 & 0.116 & 0.228 & 0.251 & 0.11 \\
\hline Relative value & $0.602^{* *}$ & $0.262^{* *}$ & $0.136^{* *}$ & 0.551 & 0.331 & 0.118 & 0.259 & 0.34 \\
\hline S\&P 500 & 0.923 & 0.089 & -0.012 & 1.000 & 0.000 & 0.000 & 1.000 & 1.00 \\
\hline
\end{tabular}

Significance levels are denoted by ${ }^{* *}$ for $1 \%$ and ${ }^{*}$ for $5 \%$. 
Table A.1

Monte Carlo simulations: Descriptive statistics of the estimates of the market exposure and the smoothing coefficients

The table reports the true values and some descriptive statistics of the distributions of their estimates of the market exposure $(\beta$. $)$ and the smoothing coefficients $\left(\theta_{., .}\right)$of the Equity Hedge and Event-Driven indices. The calculations are based on ordinary least squares regressions on 5,000 random samples designed to mimic the behavior of the negative tails of the returns of the Standard and Poor's 500 index and the idiosyncratic components of the returns of the hedge fund indices. The true values of the parameters for the two hedge fund indices are set equal to our estimates at the end of the sample (cf. Figure A.2).

\begin{tabular}{cccccccc}
\hline Parameter & $\begin{array}{c}\text { True } \\
\text { Value }\end{array}$ & Mean & $\begin{array}{c}5^{\text {th }} \\
\text { percentile }\end{array}$ & $\begin{array}{c}10^{\text {th }} \\
\text { percentile }\end{array}$ & $\begin{array}{c}90^{\text {th }} \\
\text { percentile }\end{array}$ & $\begin{array}{c}95^{\text {th }} \\
\text { percentile }\end{array}$ & $\begin{array}{c}\text { Mean } \\
\text { absolute } \\
\text { error }\end{array}$ \\
\hline$\beta_{1}$ & 0.61 & 0.619 & 0.464 & 0.498 & 0.743 & 0.790 & 0.078 \\
$\theta_{1,1}$ & 0.79 & 0.790 & 0.644 & 0.678 & 0.910 & 0.949 & 0.072 \\
$\theta_{1,2}$ & 0.10 & 0.102 & 0.000 & 0.000 & 0.187 & 0.216 & 0.054 \\
$\theta_{1,3}$ & 0.11 & 0.109 & 0.000 & 0.003 & 0.194 & 0.223 & 0.054 \\
$\beta_{2}$ & 0.53 & 0.532 & 0.394 & 0.429 & 0.635 & 0.672 & 0.065 \\
$\theta_{2,1}$ & 0.67 & 0.677 & 0.562 & 0.588 & 0.775 & 0.818 & 0.059 \\
$\theta_{2,2}$ & 0.18 & 0.176 & 0.072 & 0.104 & 0.242 & 0.263 & 0.043 \\
$\theta_{2,3}$ & 0.15 & 0.147 & 0.035 & 0.070 & 0.217 & 0.240 & 0.046 \\
\hline
\end{tabular}

Table A.2

Monte Carlo simulations: Bias and mean absolute error of the estimates of the Value-at-Risk (VaR) and extreme linkage measure (ELM)

The table reports the bias and the mean absolute error of the estimates of VaR of the Equity Hedge index and ELM between the Equity Hedge and Event-Driven indices, for a few values of the size of the sample. The calculations are based on 5,000 random samples designed to mimic the behavior of the negative tails of the returns of the Standard and Poor's 500 index and the idiosyncratic components of the returns of the hedge fund indices. In the simulations, we set the values of the market exposure and the smoothing coefficients for the two indices equal to our estimates at the end of the sample (cf. Fig. A.2). Based on our estimates for the market exposures and the smoothing coefficients, the true values of the risk measures are $\mathrm{VaR}=11.7$ and ELM $=0.39$ (cf. Fig. 5, Panel A, and Fig. 7).

\begin{tabular}{|c|c|c|c|c|}
\hline \multirow{2}{*}{$\begin{array}{l}\text { Number of } \\
\text { observations }\end{array}$} & \multicolumn{2}{|c|}{ VaR } & \multicolumn{2}{|c|}{ ELM } \\
\hline & Bias & $\begin{array}{c}\text { Mean } \\
\text { absolute error }\end{array}$ & Bias & $\begin{array}{c}\text { Mean } \\
\text { absolute error }\end{array}$ \\
\hline 60 & 0.181 & 1.019 & -0.046 & 0.110 \\
\hline 150 & 0.055 & 0.658 & -0.014 & 0.080 \\
\hline 300 & 0.019 & 0.468 & -0.002 & 0.064 \\
\hline
\end{tabular}




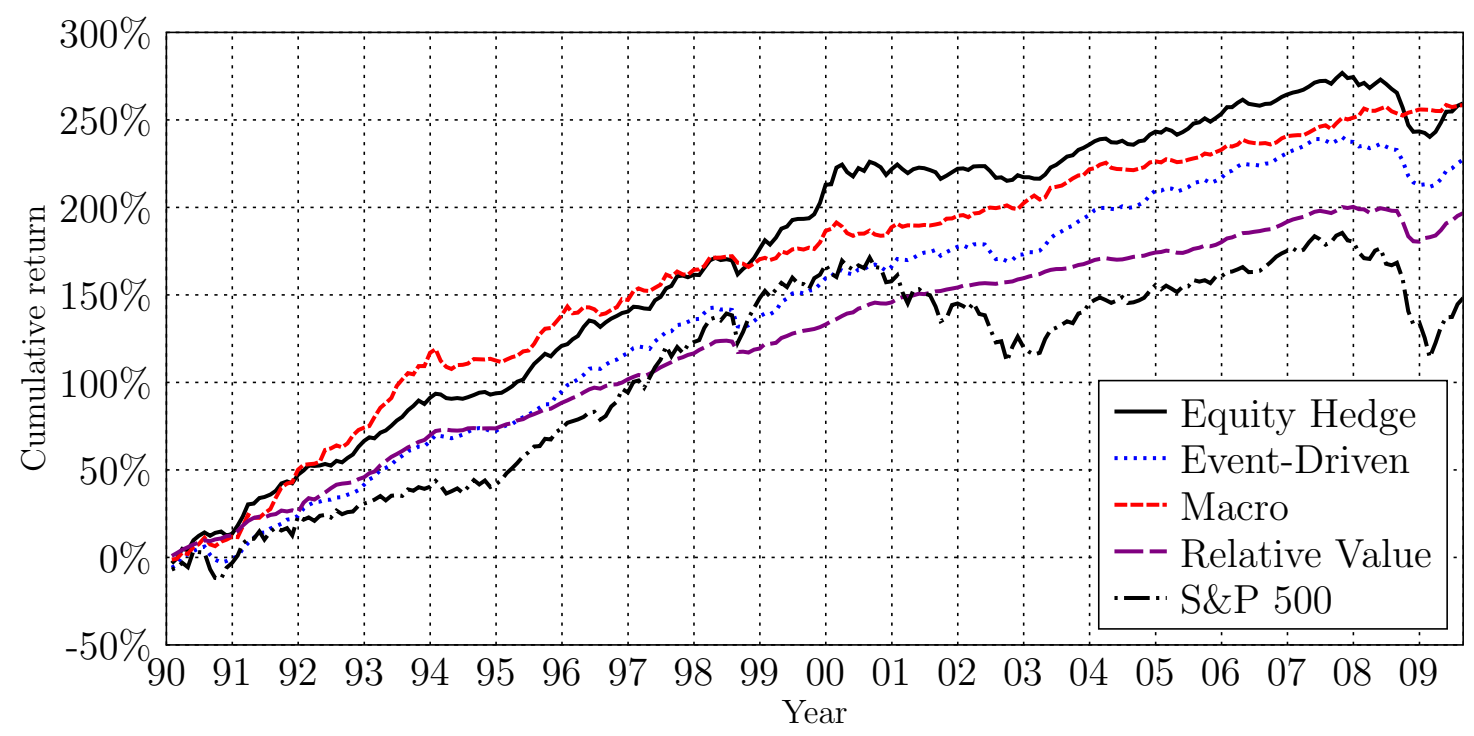

Fig. 1. Cumulative monthly log-returns of four hedge fund indices for the period January 1990 to August 2009. 

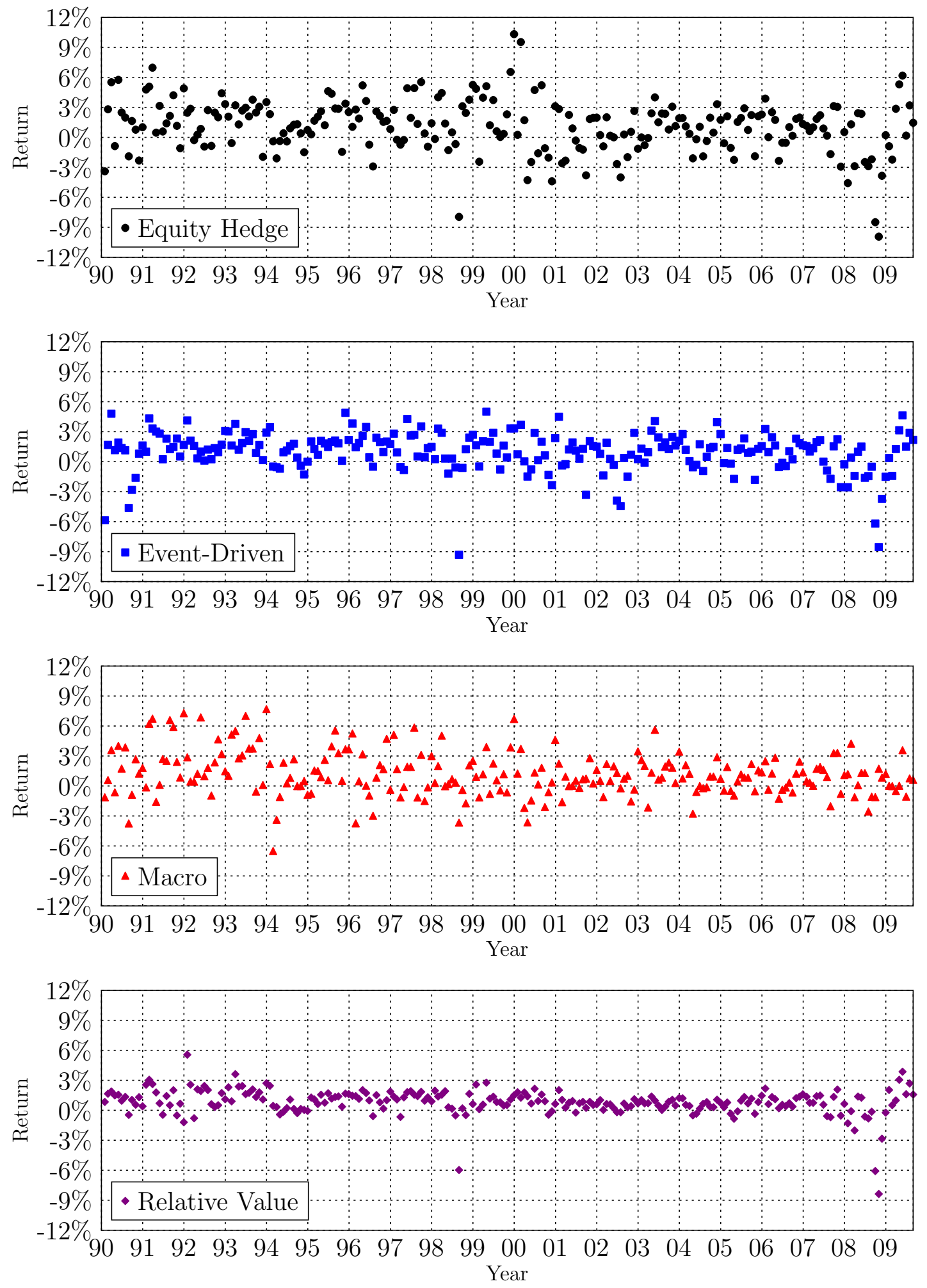

Fig. 2. Monthly log-returns of four hedge fund indices for the period January 1990 to August 2009 . 


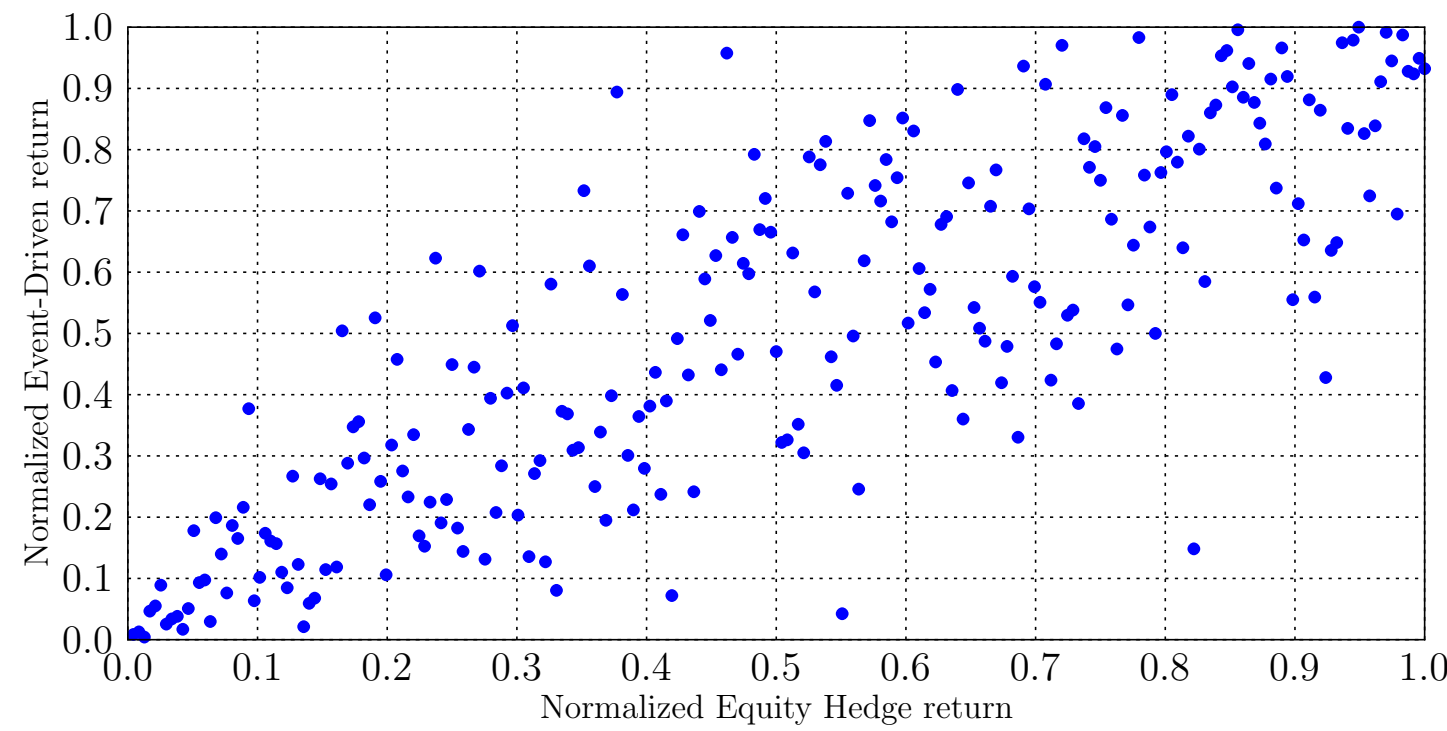

Fig. 3. Normalized monthly returns of two hedge funds indices. Scatter plot of the pairs $\left(y_{1, t}, y_{2, t}\right)_{t=1, \ldots, 236}$, where $y_{1, t}=\hat{F}_{X_{1}}\left(x_{1, t}\right), y_{2, t}=\hat{F}_{X_{2}}\left(x_{2, t}\right)$, and $\hat{F}_{X_{1}}$ and $\hat{F}_{X_{2}}$ are the empirical cumulative distribution functions of the monthly log-returns of the Equity Hedge and Event-Driven indices for the period January 1990 to August 2009. The plot shows the empirical joint distribution of the returns of the two indices that have been normalized to have uniform marginal distributions. 

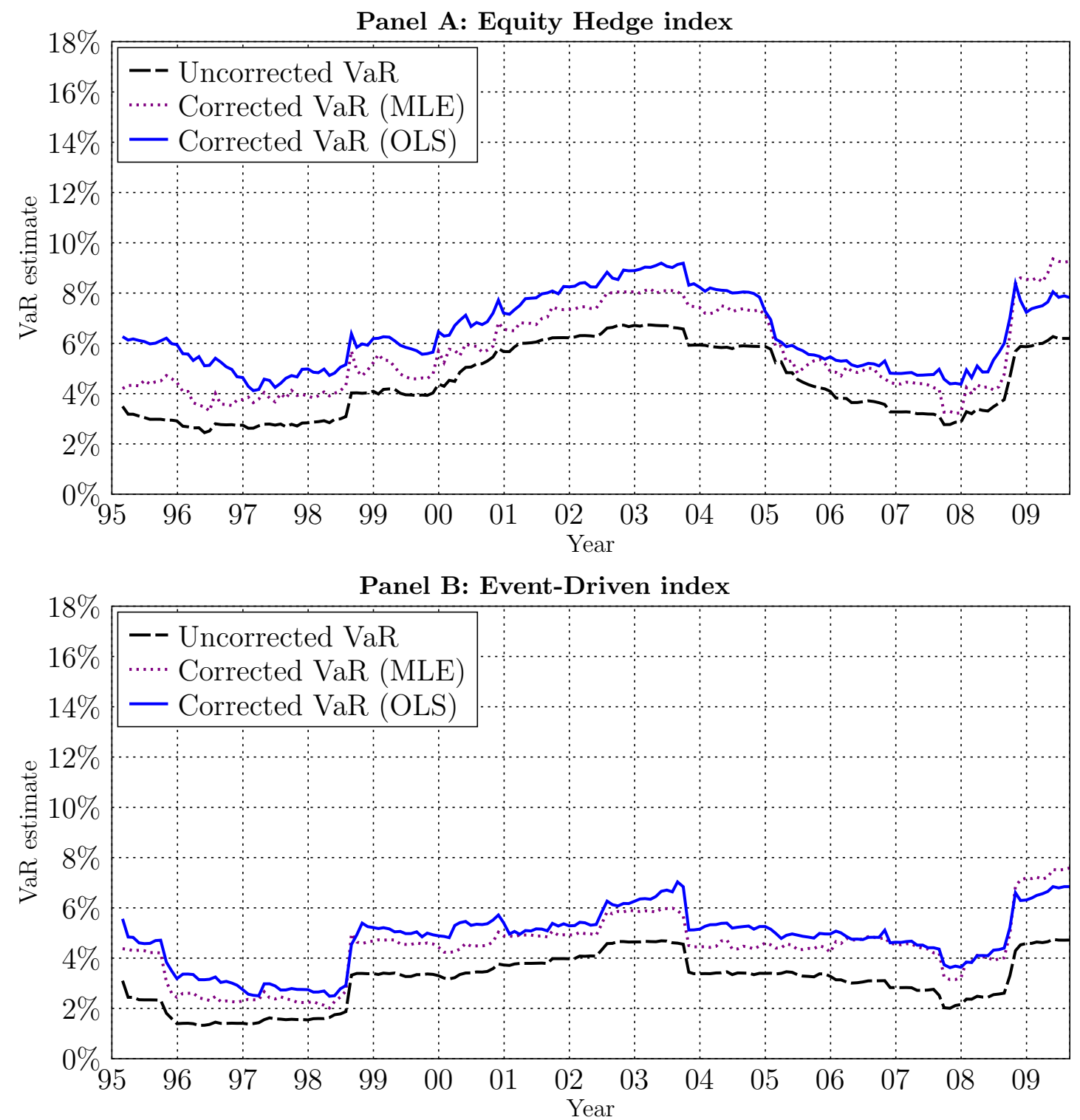

Fig. 4. The Value-at-Risk (VaR) for the Equity Hedge (Panel A) and Event-Driven (Panel B) indices at the $1 \%$ confidence level under the assumption of normally distributed monthly returns. The uncorrected VaR, which is based on reported returns, and the corrected VaR, which is based on the true unobservable returns, are equal, respectively, to $\sigma_{S} \Phi^{-1}(1-p)+\mu_{S}$ and $\sigma_{X} \Phi^{-1}(1-p)+\mu_{S}$, where $p$ is the confidence level, $\sigma_{S}$ is the volatility of the reported returns, $\mu_{S}$ is the mean of the reported returns, and $\sigma_{X}=\sigma_{S}\left(\sum_{k=0}^{2} \theta_{k}^{2}\right)^{1 / 2}$. The maximum likelihood estimate (MLE) of the smoothing coefficients $\left(\theta_{k}\right)$ is based on the model $S_{t}=\sum_{k=0}^{2} \theta_{k} X_{t-k}$ in which returns reported by hedge funds $\left(S_{t}\right)$ are weighted averages of current and past unobservable returns $\left(X_{t}\right)$ that are assumed to be independent and identically distributed (i.i.d.). The linear regression estimates (ordinary least squares [OLS]) make the additional assumption that $X_{t}=\beta R_{t}+\varepsilon_{t}$, where $R_{t}$ is the market return and $\varepsilon_{t}$ is an i.i.d. idiosyncratic risk factor. In both cases, the smoothing coefficients are assumed to satisfy the constraints $\theta_{k} \in[0,1]$, for $k=0,1,2$, and $\sum_{k=0}^{2} \theta_{k}=1$. In the OLS estimation, the Standard and Poor's 500 total return index is used as market factor. The estimations are based on rolling windows of 60 months ending in the reference month. 

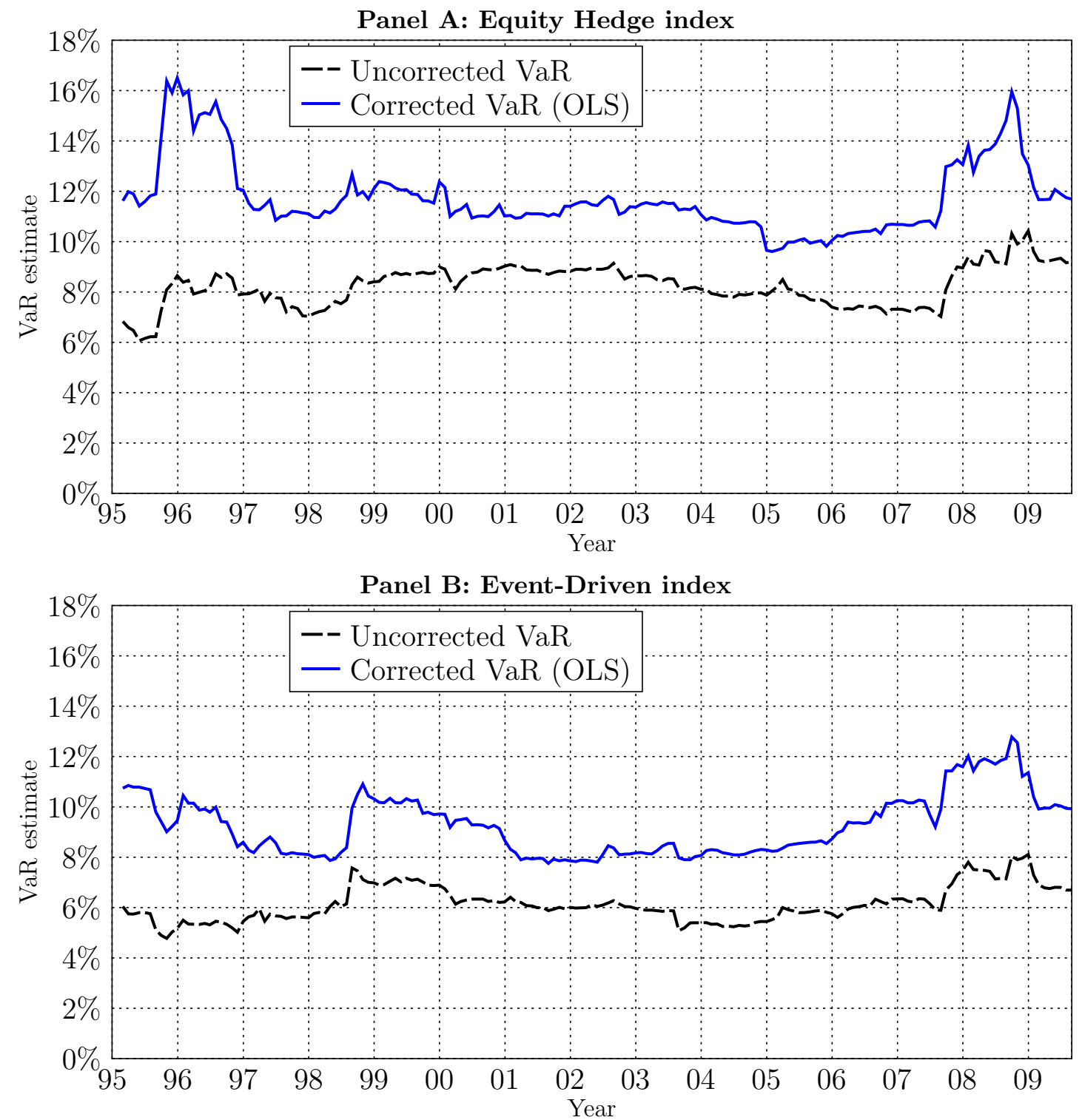

Fig. 5. The Value-at-Risk (VaR) for the Equity Hedge (Panel A) and Event-Driven (Panel B) indices at the $1 \%$ confidence level under the assumption of fat tailed distributed monthly returns. The uncorrected VaR, which is based on reported returns, and the corrected VaR, which is based on the true unobservable returns, are equal, respectively, to $\left(\left(\gamma_{R} \beta^{\alpha}+\gamma_{\varepsilon}\right)\left(\sum_{k=0}^{2} \theta_{k}^{\alpha}\right) / p\right)^{1 / \alpha}$ and $\left(\left(\gamma_{R} \beta^{\alpha}+\gamma_{\varepsilon}\right) / p\right)^{1 / \alpha}$, where $p$ is the confidence level. The smoothing coefficients $\left(\theta_{k}\right)$ and the market exposure $(\beta)$ are estimated by linear regressions (ordinary least squares [OLS]) of the model $S_{t}=\sum_{k=0}^{2} \theta_{k} X_{t-k}$ in which returns reported by hedge funds $\left(S_{t}\right)$ are weighted averages of current and past unobservable returns $\left(X_{t}\right)$. Unobservable returns are assumed to be of the form $X_{t}=\beta R_{t}+\varepsilon_{t}$, where $R_{t}$ is the market return and $\varepsilon_{t}$ is an independent and identically distributed idiosyncratic risk factor. The smoothing coefficients are assumed to satisfy the constraints $\theta_{k} \in[0,1]$, for $k=0,1,2$, and $\sum_{k=0}^{2} \theta_{k}=1$. The estimations use the Standard and Poor's 500 total return index as market factor and are based on rolling windows of 60 months ending in the reference month. The tail index $(\alpha)$ and the scale parameter of the market factor $\left(\gamma_{R}\right)$, estimated using Hill plots, are kept fixed at 3 and 48.0, respectively. The scale parameters $\left(\gamma_{\varepsilon}\right)$ of the idiosyncratic terms of the Equity Hedge and Event-Driven indices are estimated using Hill plots and kept fixed at 5.0 and 2.6 , respectively. 


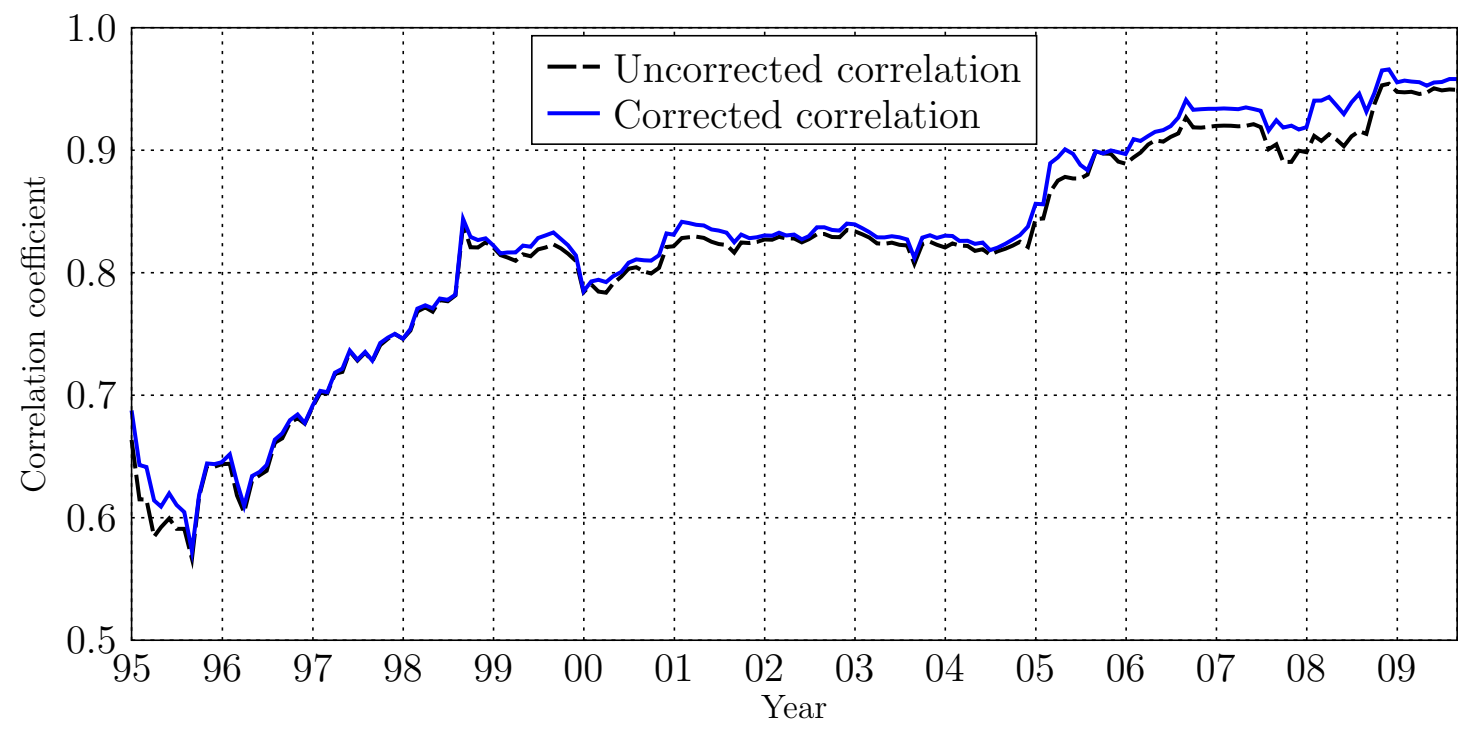

Fig. 6. Correlation between the Equity Hedge and Event-Driven indices. The uncorrected correlation is the usual Pearson's correlation between reported returns whereas the corrected correlation, which is based on the true unobservable returns, is adjusted by the factor $\left(\sum_{k=0}^{2} \theta_{1, k} \theta_{2, k}\right) /\left(\left(\sum_{k=0}^{2} \theta_{1, k}^{2}\right)\left(\sum_{k=0}^{2} \theta_{2, k}^{2}\right)\right)^{1 / 2}$. The smoothing coefficients $\left(\theta_{1, k}\right)$ and $\left(\theta_{2, k}\right)$ are estimated by a maximum likelihood estimation of the model $S_{t}=\sum_{k=0}^{2} \theta_{k} X_{t-k}$ in which returns reported by hedge funds $\left(S_{t}\right)$ are weighted averages of current and past unobservable returns $\left(X_{t}\right)$. Unobservable returns are assumed to be independent and identically distributed and the smoothing coefficients are assumed to satisfy the constraints $\theta_{k} \in[0,1]$, for $k=0,1,2$, and $\sum_{k=0}^{2} \theta_{k}=1$. The estimations are based on rolling windows of 60 months ending in the reference month. 


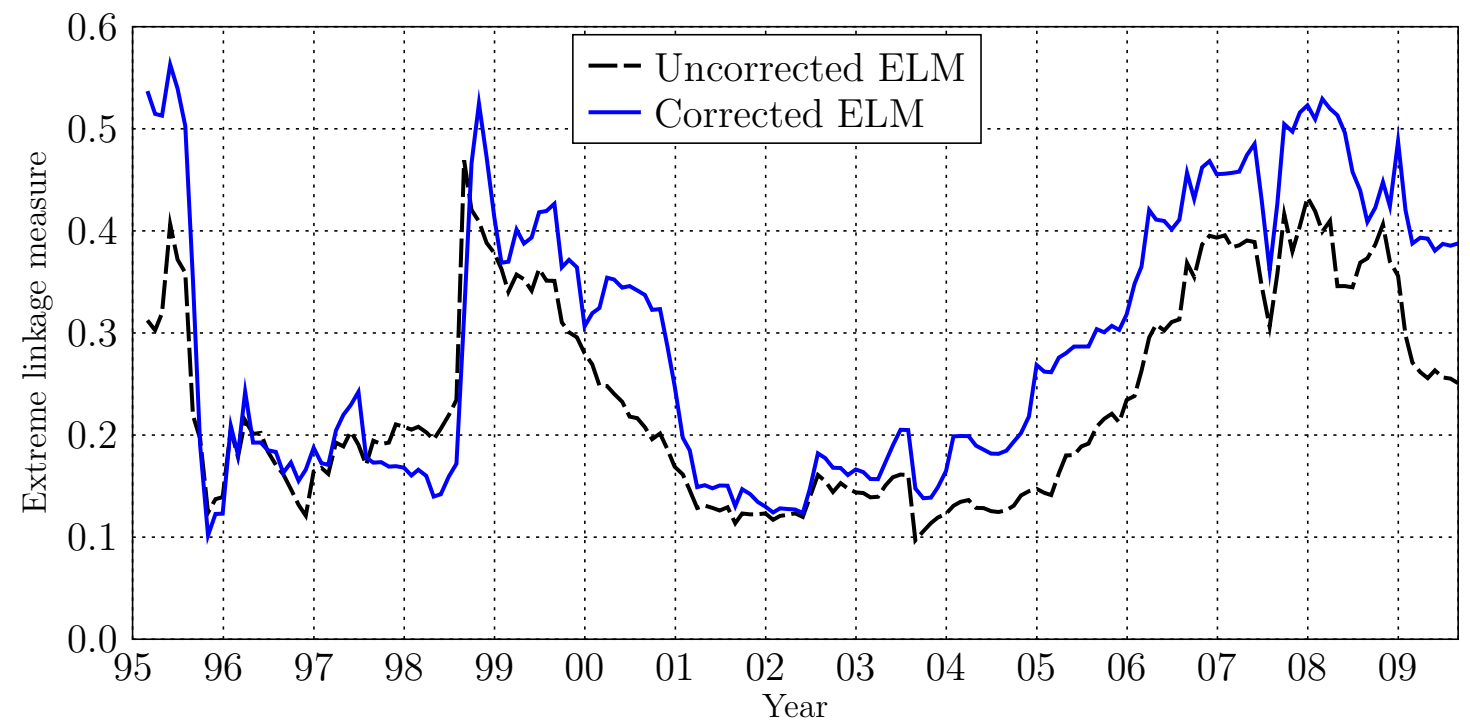

Fig. 7. Extreme linkage measure (ELM) between the Equity Hedge and Event-Driven indices. The uncorrected ELM, which is based on reported returns, and the corrected ELM, which is based on the true unobservable returns, are equal, respectively, to $\left(\left(\min \left(\beta_{1}, \beta_{2}\right)\right)^{\alpha} \gamma_{R}\right) /\left(\gamma_{\varepsilon_{1}}+\right.$ $\left.\gamma_{\varepsilon_{2}}+\left(\max \left(\beta_{1}, \beta_{2}\right)\right)^{\alpha} \gamma_{R}\right)$ and $\left(\sum_{k=0}^{2}\left(\min \left(\beta_{1} \theta_{1, k}, \beta_{2} \theta_{2, k}\right)\right)^{\alpha} \gamma_{R}\right) /\left(\sum_{k=0}^{2}\left(\theta_{1, k}^{\alpha} \gamma_{\varepsilon_{1}}+\theta_{2, k}^{\alpha} \gamma_{\varepsilon_{2}}+\right.\right.$ $\left.\left.\left(\max \left(\beta_{1} \theta_{1, k}, \beta_{2} \theta_{2, k}\right)\right)^{\alpha} \gamma_{R}\right)\right)$. The smoothing coefficients $\left(\theta_{k}\right)$ and the market exposure $(\beta)$ are estimated by linear regressions of the model $S_{t}=\sum_{k=0}^{2} \theta_{k} X_{t-k}$ in which returns reported by hedge funds $\left(S_{t}\right)$ are weighted averages of current and past unobservable returns $\left(X_{t}\right)$. Unobservable returns are assumed to be of the form $X_{t}=\beta R_{t}+\varepsilon_{t}$, where $R_{t}$ is the market return and $\varepsilon_{t}$ is an independent and identically distributed idiosyncratic risk factor. The smoothing coefficients are assumed to satisfy the constraints $\theta_{k} \in[0,1]$, for $k=0,1,2$, and $\sum_{k=0}^{2} \theta_{k}=1$. The estimations use the Standard and Poor's 500 total return index as market factor and are based on rolling windows of 60 months ending in the reference month. The tail index $(\alpha)$ and the scale parameter of the market factor $\left(\gamma_{R}\right)$, estimated using Hill plots, are kept fixed at 3 and 48.0, respectively. The scale parameters $\left(\gamma_{\varepsilon}\right)$ of the idiosyncratic terms of the Equity Hedge and Event-Driven indices, estimated using Hill plots, are kept fixed at 5.0 and 2.6, respectively. 

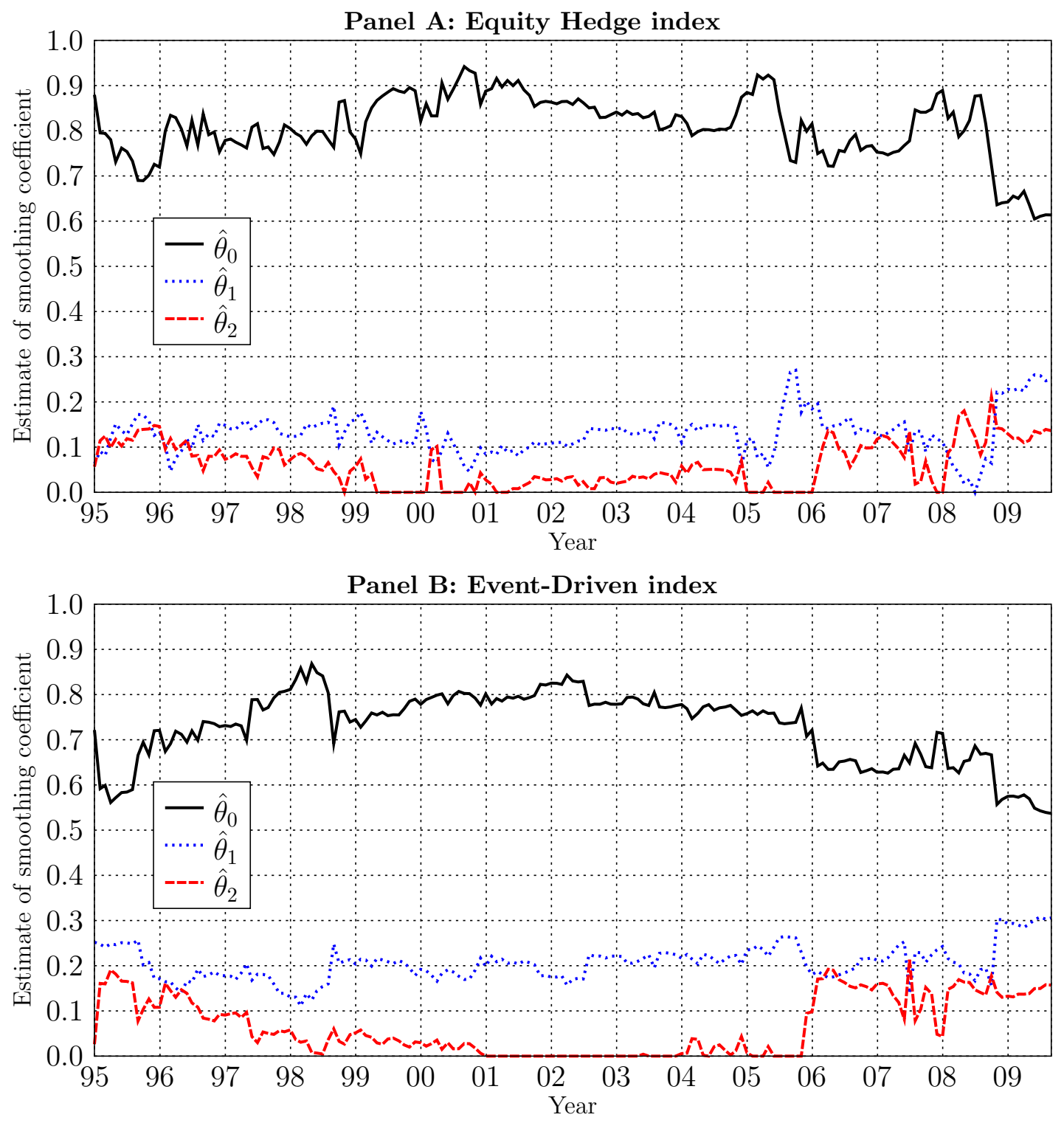

Fig. A.1. Maximum likelihood estimates for the Equity Hedge (Panel A) and Event-Driven (Panel B) indices of the smoothing coefficients $\left(\theta_{k}\right)$ of the model $S_{t}=\sum_{k=0}^{2} \theta_{k} X_{t-k}$ in which returns reported by hedge funds $\left(S_{t}\right)$ are weighted averages of current and past unobservable returns $\left(X_{t}\right)$. Unobservable returns are assumed to be independent and identically distributed and the smoothing coefficients are assumed to satisfy the constraints $\theta_{k} \in[0,1]$, for $k=0,1,2$, and $\sum_{k=0}^{2} \theta_{k}=1$. The estimations are based on rolling windows of 60 months ending in the reference month. 

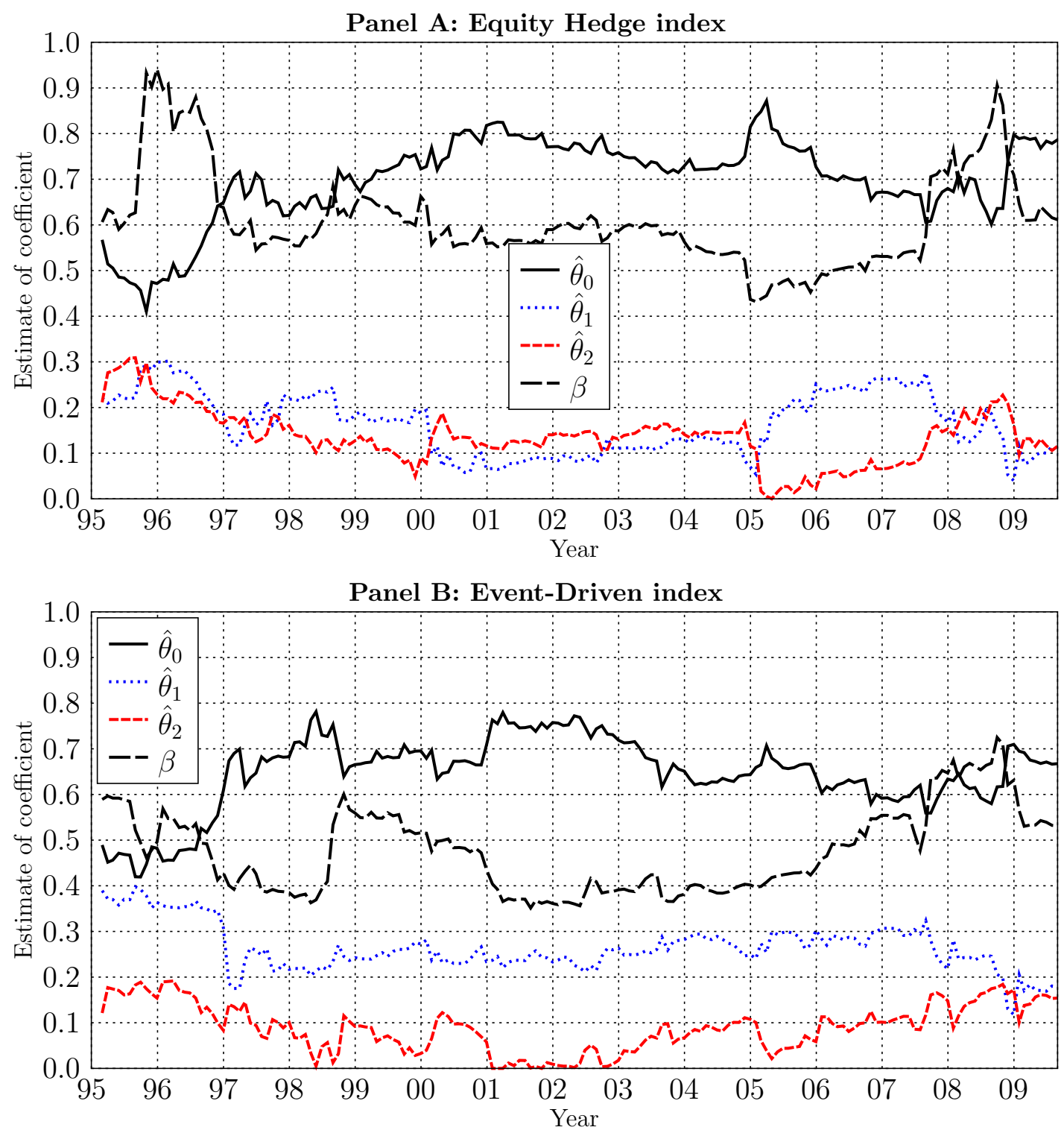

Fig. A.2. Linear regression estimates for the Equity Hedge (Panel A) and Event-Driven (Panel B) indices of the smoothing coefficients $\left(\theta_{k}\right)$ and the market exposure $(\beta)$ of the model $S_{t}=\sum_{k=0}^{2} \theta_{k} X_{t-k}$ in which returns reported by hedge funds $\left(S_{t}\right)$ are weighted averages of current and past unobservable returns $\left(X_{t}\right)$. Unobservable returns are assumed to be of the form $X_{t}=\beta R_{t}+\varepsilon_{t}$, where $R_{t}$ is the market return and $\varepsilon_{t}$ is an independent and identically distributed (i.i.d.) idiosyncratic risk factor. The smoothing coefficients are assumed to satisfy the constraints $\theta_{k} \in[0,1]$, for $k=0,1,2$, and $\sum_{k=0}^{2} \theta_{k}=1$. As suggested by Getmansky et al. (2004), we obtain consistent estimates of the parameters by running ordinary least squares (OLS) regressions of the equation $S_{t}=\mu+\beta\left(\theta_{0} R_{t}+\theta_{1} R_{t-1}+\theta_{2} R_{t-2}\right)+u_{t}=\mu+\gamma_{0} R_{t}+\gamma_{1} R_{t-1}+\gamma_{2} R_{t-2}+u_{t}$, where $u_{t}=\theta_{0} \varepsilon_{t}+\theta_{1} \varepsilon_{t-1}+\theta_{2} \varepsilon_{t-2}$ and $\varepsilon_{t}$ is i.i.d. In this case, $\hat{\beta}=\hat{\gamma}_{0}+\hat{\gamma}_{1}+\hat{\gamma}_{2}$ and $\hat{\theta}_{k}=\hat{\gamma}_{k} / \hat{\beta}$. To have efficient estimates, Getmansky et al. (2004) also suggest using a maximum likelihood estimator. In our case the results are almost identical for both OLS and maximum likelihood estimators. The estimations use the Standard and Poor's 500 total return index as market factor and are based on rolling windows of 60 months ending in the reference month. 

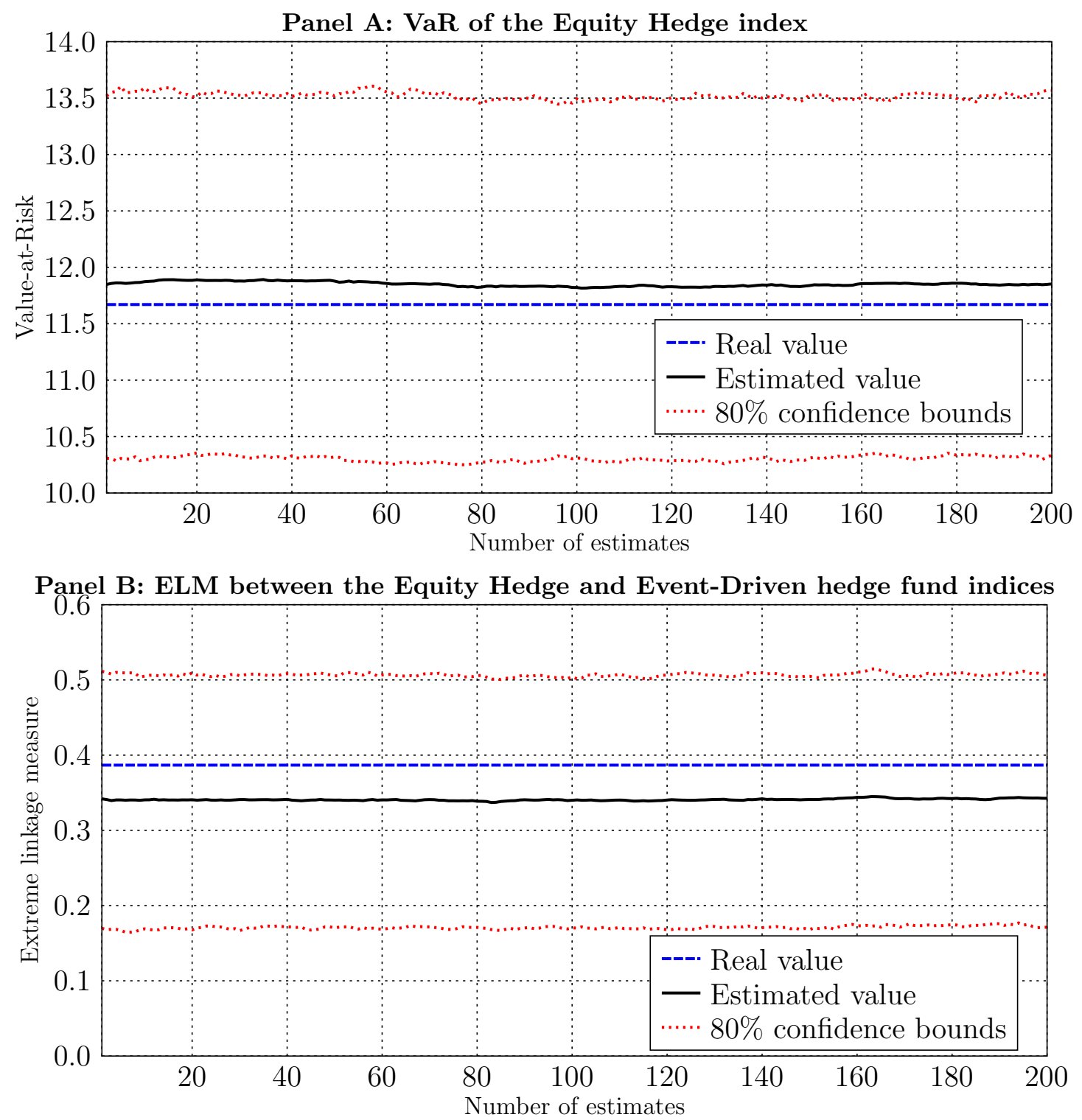

Fig. A.3. Monte Carlo simulations: Bias and confidence intervals of the estimates of the Valueat-Risk (VaR; Panel A) of the Equity Hedge index and of the extreme linkage measure (ELM; Panel B) between the Equity Hedge and Event-Driven hedge fund indices. The figure shows the mean values, and the real values as well, of the estimates of VaR and ELM based on 200 moving windows of 60 observations of 5,000 random samples of 261 datapoints. The random samples are designed to mimic the behavior of the negative tails of the returns of the Standard and Poor's 500 index and the idiosyncratic components of the returns of the Equity Hedge and Event-Driven hedge fund indices. In the simulations we set the values of the market exposure and the smoothing coefficients for the two indices equal to our estimates at the end of the sample (cf. Fig. A.2). Based on our estimates for the market exposures and the smoothing coefficients, the true values of the risk measures are VaR $=11.7$ and ELM $=0.39$ (cf. Figs. 5 and 7 ). The dotted lines represent $80 \%$ confidence intervals. 